\title{
Three lectures on Algebraic Microlocal Analysis.
}

Spring school, Northwestern University, May 2012

Pierre Schapira 


\section{Contents}

1 Microlocalization 5

1.1 Generalized functions . . . . . . . . . . . . . 5

1.2 Microlocalization . . . . . . . . . . . . . . 7

1.3 Microsupport . . . . . . . . . . . . . . . . . . 12

1.4 The functor hhom . . . . . . . . . . . . . . . 13

1.5 An application: elliptic pairs . . . . . . . . . . . 14

2 Microlocal Euler classes

2.1 Complex manifolds . . . . . . . . . . . . . . . . 17

2.2 Sheaves . . . . . . . . . . . . . . . . . . . . 20

2.3 Microlocal Euler classes . . . . . . . . . . . . . . . 23

2.4 Constructible sheaves . . . . . . . . . . . . . . . . . . . . . . . . . . . . . .

2.5 D-modules . . . . . . . . . . . . . . . . . . . . . . . . . . . . . . . . . . . . . . . .

2.6 Elliptic pairs . . . . . . . . . . . . . . . 27

3 Indsheaves $\quad 29$

3.1 Ind-sheaves . . . . . . . . . . . . . . . . . . . . . . . 29

3.2 Sheaves on the subanalytic site . . . . . . . . . . . . . . . . . . . .

3.3 Moderate and formal cohomology . . . . . . . . . . . . 33

3.4 Applications to $\mathscr{D}$-modules I . . . . . . . . . . . . . . . . . 37

3.5 Applications to $\mathscr{D}$-modules II . . . . . . . . . . . . . 37 


\title{
Lecture 1
}

\section{Microlocalization of sheaves}

\begin{abstract}
This first talk is a survey talk with some historical comments and I refer to [Sc10] for a more detailed overview.

I will first explain the notions of Sato's hyperfunctions and microfunctions, at the origin of the story, and I will describe the Sato's microlocalization functor which was first motivated by problems of Analysis (see [SKK73]). Then I will briefly recall the main features of the microlocal theory of sheaves of [KS90] with emphasize on the functor $\mu$ hom which will be the main tool for the second talk.
\end{abstract}

\subsection{Generalized functions}

In the sixties, people were used to work with various spaces of generalized functions constructed with the tools of functional analysis. Sato's construction of hyperfunctions in 59-60 is at the opposite of this practice: he uses purely algebraic tools and complex analysis. The importance of Sato's definition is twofold: first, it is purely algebraic (starting with the analytic object $\mathscr{O}_{X}$ ), and second it highlights the link between real and complex geometry. (See [Sa60] and see [Sc07] for an exposition of Sato's work.)

Consider first the case where $M$ is an open subset of the real line $\mathbb{R}$ and let $X$ be an open neighborhood of $M$ in the complex line $\mathbb{C}$ satisfying $X \cap \mathbb{R}=M$. The space $\mathscr{B}(M)$ of hyperfunctions on $M$ is given by

$$
\mathscr{B}(M)=\mathscr{O}(X \backslash M) / \mathscr{O}(X)
$$

It is easily proved, using the solution of the Cousin problem, that this space depends only on $M$, not on the choice of $X$, and that the correspondence $U \mapsto \mathscr{B}(U)(U$ open in $M)$ defines a flabby sheaf $\mathscr{B}_{M}$ on $M$. 
With Sato's definition, the boundary values always exist and are no more a limit in any classical sense.

Example 1.1.1. (i) The Dirac function at 0 is

$$
\delta(0)=\frac{1}{2 i \pi}\left(\frac{1}{x-i 0}-\frac{1}{x+i 0}\right) .
$$

Indeed, if $\varphi$ is a $C^{0}$-function on $\mathbb{R}$ with compact support, one has

$$
\varphi(0)=\lim _{\varepsilon \geq_{0}} \frac{1}{2 i \pi} \int_{\mathbb{R}}\left(\frac{\varphi(x)}{x-i \varepsilon}-\frac{\varphi(x)}{x+i \varepsilon}\right) d x .
$$

(ii) The holomorphic function $\exp (1 / z)$ defined on $\mathbb{C} \backslash\{0\}$ has a boundary value as a hyperfunction (supported by $\{0\}$ ) not as a distribution.

On a real analytic manifold $M$ of dimension $n$, the sheaf $\mathscr{B}_{M}$ was originally defined as

$$
\mathscr{B}_{M}=H_{M}^{n}\left(\mathscr{O}_{X}\right) \otimes \text { or }_{M}
$$

where $X$ is a complexification of $M$ and or $_{M}$ is the orientation sheaf on $M$. It is shown that this object is concentrated in degree 0 . Since $X$ is oriented, Poincaré's duality gives the isomorphism $\mathrm{D}_{X}^{\prime}\left(\mathbb{C}_{M}\right) \simeq \operatorname{or}_{M}[-n]$ (see (1.2.1) below for the definition of $\left.\mathrm{D}_{M}^{\prime}\right)$. An equivalent definition of hyperfunctions is thus given by

$$
\mathscr{B}_{M}=\mathrm{R} \mathscr{H} o m_{\mathbb{C}_{X}}\left(\mathrm{D}_{X}^{\prime}\left(\mathbb{C}_{M}\right), \mathscr{O}_{X}\right)
$$

Let us define the notion of "boundary value" in this settings. Consider a subanalytic open subset $\Omega$ of $X$ and denote by $\bar{\Omega}$ its closure. Assume that:

$$
\left\{\begin{array}{l}
\mathrm{D}_{X}^{\prime}\left(\mathbb{C}_{\Omega}\right) \simeq \mathbb{C}_{\bar{\Omega}} \\
M \subset \bar{\Omega}
\end{array}\right.
$$

The morphism $\mathbb{C}_{\bar{\Omega}} \rightarrow \mathbb{C}_{M}$ defines by duality the morphism $\mathrm{D}_{X}^{\prime}\left(\mathbb{C}_{M}\right) \rightarrow$ $\mathrm{D}_{X}^{\prime}\left(\mathbb{C}_{\bar{\Omega}}\right) \simeq \mathbb{C}_{\Omega}$. Applying the functor $\operatorname{RHom}\left(\cdot, \mathscr{O}_{X}\right)$, we get the boundary value morphism

$$
\mathrm{b}: \mathscr{O}(\Omega) \rightarrow \mathscr{B}(M)
$$

When considering operations on hyperfunctions such as integral transforms, one is naturally lead to consider more general sheaves of generalized functions such as R $\mathscr{H} o m\left(G, \mathscr{O}_{X}\right)$ where $G$ is a constructible sheaf. We shall come back on this point later. 
Similarly as in dimension one, we can represent the sheaf $\mathscr{B}_{M}$ by using Čech cohomology of coverings of $X \backslash M$. For example, let $X$ be a Stein open subset of $\mathbb{C}^{n}$ and set $M=\mathbb{R}^{n} \cap X$. Denote by $x$ the coordinates on $\mathbb{R}^{n}$ and by $x+i y$ the coordinates on $\mathbb{C}^{n}$. One can recover $\mathbb{C}^{n} \backslash \mathbb{R}^{n}$ by $n+1$ open half-spaces $V_{i}=\left\langle y, \xi_{i}\right\rangle>0(i=1, \ldots, n+1)$. For $J \subset\{1, \ldots, n+1\}$ set $V_{J}=\bigcap_{j \in J} V_{j}$. Assuming $n>1$, we have the isomorphism $H_{M}^{n}\left(X ; \mathscr{O}_{X}\right) \simeq H^{n-1}\left(X \backslash M ; \mathscr{O}_{X}\right)$. Therefore, setting $U_{J}=V_{J} \cap X$, one has

$$
\mathscr{B}(M) \simeq \sum_{|J|=n} \mathscr{O}_{X}\left(U_{J}\right) / \sum_{|K|=n-1} \mathscr{O}_{X}\left(U_{K}\right)
$$

On a real analytic manifold $M$, any hyperfunction $u \in \Gamma(M ; \mathscr{B})$ is a (non unique) sum of boundary values of holomorphic functions defined in tubes with edge $M$. Such a decomposition leads to the so-called Edge of the Wedge theorem and was intensively studied in the seventies (see [Mr67, BI73]).

Then comes naturally the following problem: how to recognize the directions associated with these tubes? The answer is given by the Sato's microlocalization functor.

\subsection{Microlocalization}

Unless otherwise specified, all manifolds are real, say of class $C^{\infty}$ and $\mathbf{k}$ denotes a commutative unital ring with finite global homological dimension.

We denote by $\mathbf{k}_{M}$ the constant sheaf on $M$ with stalk $\mathbf{k}$, by $\mathrm{D}^{\mathrm{b}}\left(\mathbf{k}_{M}\right)$ the bounded derived category of sheaves of k-modules on $M$ and by $\mathrm{D}_{\mathrm{cc}}^{\mathrm{b}}\left(\mathbf{k}_{M}\right)$ the full triangulated subcategory of $\mathrm{D}^{\mathrm{b}}\left(\mathbf{k}_{M}\right)$ consisting of cohomologically constructible objects. If $M$ is real analytic, we denote by $\mathrm{D}_{\mathbb{R}-\mathrm{c}}^{\mathrm{b}}\left(\mathbf{k}_{M}\right)$ the triangulated category of $\mathbb{R}$-constructible sheaves.

We denote by $\omega_{M}$ the dualizing complex on $M$. Then $\omega_{M} \simeq \operatorname{or}_{M}[\operatorname{dim} M]$ where or $_{M}$ is the orientation sheaf and $\operatorname{dim} M$ the dimension of $M$. We shall use the duality functors

$$
\mathrm{D}_{M}^{\prime} F=\mathrm{R} \mathscr{H} o m\left(F, \mathbf{k}_{M}\right), \quad \mathrm{D}_{M} F=\mathrm{R} \mathscr{H} o m\left(F, \omega_{M}\right) .
$$

For a locally closed subset $A$ of $M$, we denote by $\mathbf{k}_{M A}$ the sheaf which is the constant sheaf on $A$ with stalk $\mathbf{k}$ and which is 0 on $M \backslash A$. If there is no risk of confusion, we simply denote it by $\mathbf{k}_{A}$.

\section{Fourier-Sato transform}

The classical Fourier transform interchanges (generalized) functions on a vector space $V$ and (generalized) functions on the dual vector space $V^{*}$. The 
idea of extending this formalism to sheaves, hence to replacing an isomorphism of spaces with an equivalence of categories, seems to have appeared first in Mikio Sato's construction of microfunctions in the 70's.

Let $\tau: E \rightarrow M$ be a finite dimensional real vector bundle over a real manifold $M$ with fiber dimension $n$ and let $\pi: E^{*} \rightarrow M$ be the dual vector bundle. Denote by $p_{1}$ and $p_{2}$ the first and second projection defined on $E \times_{M} E^{*}$, and define:

$$
\begin{aligned}
& P=\left\{(x, y) \in E \times_{M} E^{*} ;\langle x, y\rangle \geq 0\right\}, \\
& P^{\prime}=\left\{(x, y) \in E \times_{M} E^{*} ;\langle x, y\rangle \leq 0\right\} .
\end{aligned}
$$

Consider the diagram:

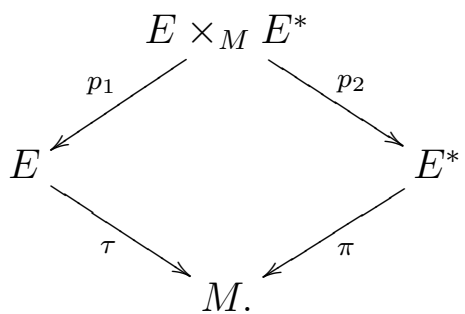

Denote by $\mathrm{D}_{\mathbb{R}^{+}}^{\mathrm{b}}\left(\mathbf{k}_{E}\right)$ the full triangulated subcategory of $\mathrm{D}^{\mathrm{b}}\left(\mathbf{k}_{E}\right)$ consisting of conic sheaves, that is, objects with locally constant cohomology on the orbits of $\mathbb{R}^{+}$.

Definition 1.2.1. Let $F \in \mathrm{D}_{\mathbb{R}^{+}}^{\mathrm{b}}\left(\mathbf{k}_{E}\right), G \in \mathrm{D}_{\mathbb{R}^{+}}^{\mathrm{b}}\left(\mathbf{k}_{E^{*}}\right)$. One sets:

$$
\begin{aligned}
F^{\wedge} & =\mathrm{R} p_{2 !}\left(p_{1}^{-1} F\right)_{P^{\prime}} \simeq \mathrm{R} p_{2 *}\left(\mathrm{R} \Gamma_{P} p_{1}^{-1} F\right) \\
G^{\vee} & =\mathrm{R} p_{1_{*}}\left(\mathrm{R} \Gamma_{P^{\prime}} p_{2}^{!} G\right) \simeq \mathrm{R} p_{1 !}\left(p_{2}^{!} G\right)_{P} .
\end{aligned}
$$

The main result of the theory is the following.

Theorem 1.2.2. The two functors $(\cdot)^{\wedge}$ and $(\cdot)^{\vee}$ are inverse to each other, hence define an equivalence of categories $\mathrm{D}_{\mathbb{R}^{+}}^{\mathrm{b}}\left(\mathbf{k}_{E}\right) \simeq \mathrm{D}_{\mathbb{R}^{+}}^{\mathrm{b}}\left(\mathbf{k}_{E^{*}}\right)$.

Example 1.2.3. (i) Let $\gamma$ be a closed proper convex cone in $E$ with $M \subset \gamma$. Then:

$$
\left(\mathbf{k}_{\gamma}\right)^{\wedge} \simeq \mathbf{k}_{\operatorname{Int} \gamma^{\circ}}
$$

Here $\gamma^{\circ}$ is the polar cone to $\gamma$, a closed convex cone in $E^{*}$ and Int $\gamma^{\circ}$ denotes its interior.

(ii) Let $\gamma$ be an open convex cone in $E$. Then:

$$
\left(\mathbf{k}_{\gamma}\right)^{\wedge} \simeq \mathbf{k}_{\gamma^{\circ a}} \otimes \text { or }_{E^{*} / M}[-n]
$$


Here $\lambda^{a}=-\lambda$, the image of $\lambda$ by the antipodal map.

(iii) Let $(x)=\left(x^{\prime}, x^{\prime \prime}\right)$ be coordinates on $\mathbb{R}^{n}$ with $\left(x^{\prime}\right)=\left(x_{1}, \ldots, x_{p}\right)$ and $\left(x^{\prime \prime}\right)=\left(x_{p+1}, \ldots, x_{n}\right)$. Denote by $(y)=\left(y^{\prime}, y^{\prime \prime}\right)$ the dual coordinates on $\left(\mathbb{R}^{n}\right)^{*}$. Set

$$
\gamma=\left\{x ; x^{\prime 2}-x^{\prime \prime 2} \geq 0\right\}, \quad \lambda=\left\{y ; y^{\prime 2}-y^{\prime \prime 2} \leq 0\right\} .
$$

Then $\left(\mathbf{k}_{\gamma}\right)^{\wedge} \simeq \mathbf{k}_{\lambda}[-p]$. (See $[$ KS97].)

\section{Specialization}

Let $\iota: N \hookrightarrow M$ be the embedding of a closed submanifold $N$ of $M$. Denote by $\tau_{M}: T_{N} M \rightarrow N$ the normal bundle to $N$.

If $F$ is a sheaf on $M$, its restriction to $N$, denoted $\left.F\right|_{N}$, may be viewed as a global object, namely the direct image by $\tau_{M}$ of a sheaf $\nu_{M} F$ on $T_{N} M$, called the specialization of $F$ along $N$. Intuitively, $T_{N} M$ is the set of light rays issued from $N$ in $M$ and the germ of $\nu_{N} F$ at a normal vector $(x ; v) \in T_{N} M$ is the germ at $x$ of the restriction of $F$ along the light ray $v$.

One constructs a new manifold $\widetilde{M}_{N}$, called the normal deformation of $M$ along $N$, together with the maps

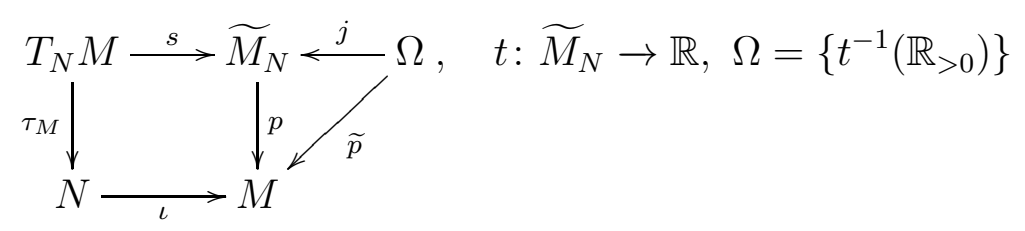

with the following properties. Locally, after choosing a local coordinate system $\left(x^{\prime}, x^{\prime \prime}\right)$ on $M$ such that $N=\left\{x^{\prime}=0\right\}$, we have $\widetilde{M}_{N}=M \times \mathbb{R}, t: \widetilde{M}_{N} \rightarrow \mathbb{R}$ is the projection, $\Omega=\{(x ; t) \in M \times \mathbb{R} ; t>0\}, p\left(x^{\prime}, x^{\prime \prime}, t\right)=\left(t x^{\prime}, x^{\prime \prime}\right)$, $T_{N} M=\{t=0\}$.

Let $S \subset M$ be a locally closed subset. The Whitney normal cone $C_{N}(S)$ is a closed conic subset of $T_{N} M$ given by

$$
C_{N}(S)=\overline{\widetilde{p}^{-1}(S)} \cap T_{N} M
$$

where, for a set $A, \bar{A}$ denotes the closure of $A$. One defines the specialization functor

$$
\nu_{N}: \mathrm{D}^{\mathrm{b}}\left(\mathbf{k}_{M}\right) \rightarrow \mathrm{D}^{\mathrm{b}}\left(\mathbf{k}_{T_{N} M}\right)
$$

by a similar formula, namely:

$$
\nu_{N} F:=s^{-1} j_{*} \widetilde{p}^{-1} F .
$$


Clearly, $\nu_{N} F \in \mathrm{D}_{\mathbb{R}^{+}}^{\mathrm{b}}\left(\mathbf{k}_{T_{N} M}\right)$, that is, $\nu_{N} F$ is a conic sheaf for the $\mathbb{R}^{+}$-action on $T_{N} M$. Moreover,

$$
\left.\left.\mathrm{R} \tau_{M *} \nu_{N} F \simeq \nu_{N} F\right|_{N} \simeq F\right|_{N} .
$$

For an open cone $V \subset T_{N} M$, one has

$$
H^{j}\left(V ; \nu_{N} F\right) \simeq \underset{U}{\lim } H^{j}(U ; F)
$$

where $U$ ranges through the family of open subsets of $M$ such that

$$
C_{N}(M \backslash U) \cap V=\emptyset .
$$

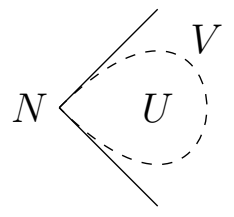

\section{Microlocalization}

Denote by $\pi_{M}: T_{N}^{*} M \rightarrow N$ the conormal bundle to $N$ in $M$, that is, the dual bundle to $\tau_{M}: T_{N} M \rightarrow N$.

If $F$ is a sheaf on $M$, the sheaf of sections of $F$ supported by $N$, denoted $\mathrm{R} \Gamma_{N} F$, may be viewed as a global object, namely the direct image by $\pi_{M}$ of a sheaf $\mu_{M} F$ on $T_{N}^{*} M$. Intuitively, $T_{N}^{*} M$ is the set of "walls" (half-spaces) in $M$ passing through $N$ and the germ of $\mu_{N} F$ at a conormal vector $(x ; \xi) \in T_{N}^{*} M$ is the germ at $x$ of the sheaf of sections of $F$ supported by closed tubes with edge $N$ and which are almost the half-space associated with $\xi$.

More precisely, the microlocalization of $F$ along $N$, denoted $\mu_{N} F$, is the Fourier-Sato transform of $\nu_{N} F$, hence is an object of $\mathrm{D}_{\mathbb{R}^{+}}^{\mathrm{b}}\left(\mathbf{k}_{T_{N}^{*} M}\right)$. It satisfies:

$$
\left.\mathrm{R} \pi_{M *} \mu_{N} F \simeq \mu_{N} F\right|_{N} \simeq \mathrm{R} \Gamma_{N} F .
$$

For a convex open cone $V \subset T_{N}^{*} M$, one has

$$
H^{j}\left(V ; \mu_{N} F\right) \simeq \underset{U, Z}{\lim _{U, Z}} H_{U \cap Z}^{j}(U ; F),
$$

where $U$ ranges over the family of open subsets of $M$ such that $U \cap N=$ $\pi_{M}(V)$ and $Z$ ranges over the family of closed subsets of $M$ such that $C_{M}(Z) \subset V^{\circ}$ where $V^{\circ}$ is the polar cone to $V$. 


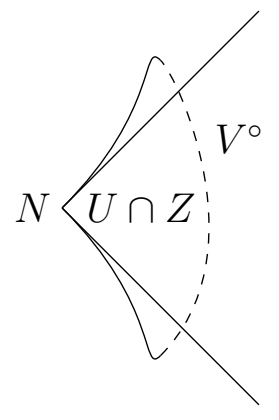

\section{Back to hyperfunctions}

Assume now that $M$ is a real analytic manifold and $X$ is a complexification of $M$. First notice the isomorphisms

$$
M \times_{X} T^{*} X \simeq \mathbb{C} \otimes_{\mathbb{R}} T^{*} M \simeq T^{*} M \oplus \sqrt{-1} T^{*} M .
$$

One deduces the isomorphism

$$
T_{M}^{*} X \simeq \sqrt{-1} T^{*} M .
$$

The sheaf $\mathscr{C}_{M}$ on $T_{M}^{*} X$ of Sato's microfunction (see [SKK73]) is defined as

$$
\mathscr{C}_{M}:=\mu_{M}\left(\mathscr{O}_{X}\right) \otimes \pi_{M}^{-1} \omega_{M}
$$

It is shown that this object is concentrated in degree 0 . Therefore, we have an isomorphism

$$
\text { spec: } \mathscr{B}_{M} \stackrel{\sim}{\longrightarrow} \pi_{M *} \mathscr{C}_{M}
$$

and Sato defines the analytic wave front set of a hyperfunction $u \in \Gamma\left(M ; \mathscr{B}_{M}\right)$ as the support of $\operatorname{spec}(u) \in \Gamma\left(T_{M}^{*} X ; \mathscr{C}_{M}\right)$.

Consider a closed convex proper cone $Z \subset T_{M}^{*} X$ which contains the zerosection $M$. Then $\operatorname{spec}(u) \subset Z$ if and only if $u$ is the boundary value of a holomorphic function defined in a tuboid $U$ with profile the interior of the polar tube to $Z^{a}$ (where $Z^{a}$ is the image of $Z$ by the antipodal map), that is, satisfying

$$
C_{M}(X \backslash U) \cap \operatorname{Int} Z^{\circ a}=\emptyset .
$$

Moreover, the sheaf $\mathscr{C}_{M}$ is conically flabby. Therefore, any hyperfunction may be decomposed as a sum of boundary values of holomorphic functions $f_{i}$ 's defined in suitable tuboids $U_{i}$ and if we have hyperfunctions $u_{i}(i=1, \ldots N)$ satisfying $\sum_{j} u_{j}=0$, there exist hyperfunctions $u_{i j}(i, j=1, \ldots N)$ such that

$$
u_{i j}=-u_{j i}, \quad u_{i}=\sum_{j} u_{i j} \text { and } \operatorname{spec}\left(u_{i j}\right) \subset \operatorname{spec}\left(u_{i}\right) \cap \operatorname{spec}\left(u_{j}\right) .
$$


When translating this result in terms of boundary values of holomorphic functions, we get the so-called "Edge of the wedge theorem", already mentioned.

Sato's introduction of the sheaf $\mathscr{C}_{M}$ was the starting point of an intense activity in the domain of linear partial differential equations after Hörmander adapted Sato's ideas to classical analysis with the help of the (usual) Fourier transform. See [Ho83] and also [BI73, Sj82 for related constructions. Note that the appearance of $\sqrt{-1}$ in the usual Fourier transform may be understood as following from the isomorphism (1.2.3).

\section{$1.3 \quad$ Microsupport}

The microsupport of sheaves has been introduced in KS82 and developed in KS85, KS90]. Roughly speaking, the microsupport of $F$ describes the codirections of non propagation of $F$. The idea of microsupport takes its origin in the study of linear PDE and particularly in the study of hyperbolic systems.

Definition 1.3.1. Let $F \in \mathrm{D}^{\mathrm{b}}\left(\mathbf{k}_{M}\right)$ and let $p \in T^{*} M$. One says that $p \notin$ $\mathrm{SS}(F)$ if there exists an open neighborhood $U$ of $p$ such that for any $x_{0} \in M$ and any real $\mathrm{C}^{1}$-function $\varphi$ on $M$ defined in a neighborhood of $x_{0}$ with $\left(x_{0} ; d \varphi\left(x_{0}\right)\right) \in U$, one has $\left(\mathrm{R} \Gamma_{\left\{x ; \varphi(x) \geq \varphi\left(x_{0}\right)\right\}} F\right)_{x_{0}} \simeq 0$.

In other words, $p \notin \mathrm{SS}(F)$ if the sheaf $F$ has no cohomology supported by "half-spaces" whose conormals are contained in a neighborhood of $p$.

- By its construction, the microsupport is $\mathbb{R}^{+}$-conic, that is, invariant by the action of $\mathbb{R}^{+}$on $T^{*} M$.

- $\mathrm{SS}(F) \cap T_{M}^{*} M=\pi_{M}(\mathrm{SS}(F))=\operatorname{Supp}(F)$.

- The microsupport satisfies the triangular inequality: if $F_{1} \rightarrow F_{2} \rightarrow$ $F_{3} \stackrel{+1}{\longrightarrow}$ is a distinguished triangle in $\mathrm{D}^{\mathrm{b}}\left(\mathbf{k}_{M}\right)$, then $\operatorname{SS}\left(F_{i}\right) \subset \operatorname{SS}\left(F_{j}\right) \cup$ $\mathrm{SS}\left(F_{k}\right)$ for all $i, j, k \in\{1,2,3\}$ with $j \neq k$.

Example 1.3.2. (i) If $F$ is a non-zero local system on $M$ and $M$ is connected, then $\operatorname{SS}(F)=T_{M}^{*} M$.

(ii) If $N$ is a closed submanifold of $M$ and $F=\mathbf{k}_{N}$, then $\operatorname{SS}(F)=T_{N}^{*} M$, the conormal bundle to $N$ in $M$.

(iii) Let $\varphi$ be a $\mathrm{C}^{1}$-function such that $d \varphi(x) \neq 0$ whenever $\varphi(x)=0$. Let $U=\{x \in M ; \varphi(x)>0\}$ and let $Z=\{x \in M ; \varphi(x) \geq 0\}$. Then

$$
\begin{aligned}
& \mathrm{SS}\left(\mathbf{k}_{U}\right)=U \times_{M} T_{M}^{*} M \cup\{(x ; \lambda d \varphi(x)) ; \varphi(x)=0, \lambda \leq 0\}, \\
& \mathrm{SS}\left(\mathbf{k}_{Z}\right)=Z \times_{M} T_{M}^{*} M \cup\{(x ; \lambda d \varphi(x)) ; \varphi(x)=0, \lambda \geq 0\} .
\end{aligned}
$$


For a precise definition of being co-isotropic (one also says involutive), we refer to [KS90, Def. 6.5.1].

Theorem 1.3.3. Let $F \in \mathrm{D}^{\mathrm{b}}\left(\mathbf{k}_{M}\right)$. Then its microsupport $\mathrm{SS}(F)$ is coisotropic.

Assume now that $\left(X, \mathscr{O}_{X}\right)$ is a complex manifold and denote as usual by $\mathscr{D}_{X}$ the sheaf of rings of finite order differential operators on $X$. For a coherent $\mathscr{D}_{X}$-module $\mathscr{M}$, one denotes by $\operatorname{char}(\mathscr{M})$ its characteristic variety, a closed conic complex analytic subvariety of $T^{*} X$. One also sets for short

$$
\operatorname{Sol}(\mathscr{M}):=\operatorname{R} \mathscr{H} o m_{\mathscr{D}}\left(\mathscr{M}, \mathscr{O}_{X}\right) .
$$

After identifying $X$ with its real underlying manifold, the link between the microsupport of sheaves and the characteristic variety of coherent $\mathscr{D}$-modules is given by

Theorem 1.3.4. Let $\mathscr{M}$ be a coherent $\mathscr{D}$-module. Then $\operatorname{SS}(\mathcal{S}$ ol $(\mathscr{M}))=$ $\operatorname{char}(\mathscr{M})$.

The inclusion $\operatorname{SS}(\mathcal{S}$ ol $(\mathscr{M})) \subset \operatorname{char}(\mathscr{M})$ is the most useful in practice. Its proof only makes use of the Cauchy-Kowalevsky theorem in its precise form given by Petrovsky and Leray (see [Ho83, § 9.4]) and of purely algebraic arguments. As a corollary of Theorems 1.3.3 and 1.3.4, one recovers the fact that the characteristic variety of a coherent $\mathscr{D}_{X}$-module is co-isotropic, a theorem of [SKK73] which also have a purely algebraic proof due to Gabber Ga81].

\subsection{The functor $\mu h o m$}

We denote by $\delta: M \rightarrow M \times M$ the diagonal embedding and we set $\Delta=\delta(M)$. For short, we also denote by $\delta$ the isomorphism

$$
\delta: T^{*} M \stackrel{\sim}{\sim} T_{\Delta}^{*}(M \times M), \quad(x ; \xi) \mapsto(x, x ; \xi,-\xi) .
$$

Let us briefly recall the main properties of the functor $\mu$ hom, a variant of Sato's microlocalization functor.

$$
\begin{aligned}
& \text { uhom: } \mathrm{D}^{\mathrm{b}}\left(\mathbf{k}_{M}\right)^{\mathrm{op}} \times \mathrm{D}^{\mathrm{b}}\left(\mathbf{k}_{M}\right) \rightarrow \mathrm{D}^{\mathrm{b}}\left(\mathbf{k}_{T^{*} M}\right), \\
& \mu h o m(G, F):=\delta^{-1} \mu_{\Delta} \mathrm{R} \mathscr{H} o m\left(q_{2}^{-1} G, q_{1}^{!} F\right)
\end{aligned}
$$

where $q_{i}(i=1,2)$ denotes the $i$-th projection on $M \times M$. Note that

$$
\begin{aligned}
& \operatorname{R} \pi_{M *} \mu h o m(G, F) \simeq \operatorname{R} \mathscr{H o m}(G, F), \\
& \mu h o m\left(\mathbf{k}_{N}, F\right) \simeq \mu_{N}(F) \text { for } N \text { a closed submanifold of } M, \\
& \operatorname{supp} \mu h o m(G, F) \subset \operatorname{SS}(G) \cap \operatorname{SS}(F), \\
& \mu h o m(G, F) \simeq \mu_{\Delta}\left(F \otimes \mathrm{L}_{M} G\right) \text { if } G \text { is constructible. }
\end{aligned}
$$


In some sense, $\mu$ hom is the sheaf of microlocal morphisms. More precisely, for $p \in T^{*} M$, we have;

$$
H^{0} \mu h o m(G, F)_{p} \simeq \operatorname{Hom}_{\mathrm{D}^{\mathrm{b}}\left(\mathbf{k}_{M} ; p\right)}(G, F)
$$

where the category $\mathrm{D}^{\mathrm{b}}\left(\mathbf{k}_{M} ; p\right)$ is the localization of $\mathrm{D}^{\mathrm{b}}\left(\mathbf{k}_{M}\right)$ by the subcategory of sheaves whose microsupport does not contain $p$.

There is an interesting phenomena which holds with $\mu$ hom and not with $\mathrm{R} \mathscr{H}$ om. Indeed, assume $M$ is real analytic. Then, although the category $\mathrm{D}_{\mathbb{R}-\mathrm{c}}^{\mathrm{b}}\left(\mathbf{k}_{M}\right)$ of constructible sheaves does not admit a Serre functor, it admits a kind of microlocal Serre functor, as shown by the isomorphism, functorial with respect to $F$ and $G$ (see [KS90, Prop. 8.4.14]):

$$
\mathrm{D}_{T^{*} M} \mu h o m(F, G) \simeq \mu h o m(G, F) \otimes \pi_{M}^{-1} \omega_{M} .
$$

This confirms the fact that to fully understand constructible sheaves, it is natural to look at them microlocally, that is, in $T^{*} M$. This is also in accordance with the "philosophy" of Mirror Symmetry which interchanges the category of coherent $\mathscr{O}_{X}$-modules on a complex manifold $X$ with the Fukaya category on a symplectic manifold $Y$. In case of $Y=T^{*} M$, the Fukaya category is equivalent to the category of $\mathbb{R}$-constructible sheaves on $M$, according to Nadler-Zaslow [Na09, NZ09] (see also [FLTZ10] for related results.)

\subsection{An application: elliptic pairs}

Denote by $\dot{T}^{*} M$ the set $T^{*} M \backslash T_{M}^{*} M$ and denote by $\dot{\pi}_{M}$ the restriction of $\pi_{M}: T^{*} M \rightarrow M$ to $\dot{T}^{*} M$. If $H \in \mathrm{D}_{\mathbb{R}^{+}}^{\mathrm{b}}\left(\mathbf{k}_{T^{*} M}\right)$ is a conic sheaf on $T^{*} M$, there is the Sato's distinguished triangle

$$
\mathrm{R} \pi_{M !} H \rightarrow \mathrm{R} \pi_{*} H \rightarrow R \dot{\pi}_{M *} H \stackrel{+1}{\longrightarrow} .
$$

Applying this result with $H=\mu h o m(G, F)$ and assuming $G$ is constructible, we get the distinguished triangle

$$
\mathrm{D}_{M}^{\prime} G \otimes F \rightarrow \operatorname{R} \mathscr{H} o m(G, F) \rightarrow R \dot{\pi}_{M *} \mu h o m(G, F) .
$$

Theorem 1.5.1. (The Petrovsky theorem for sheaves.) Assume that $G$ is constructible and $\mathrm{SS}(G) \cap \mathrm{SS}(F) \subset T_{M}^{*} M$. Then the natural morphism

$$
\operatorname{R} \mathscr{H} o m\left(G, \mathbf{k}_{M}\right) \otimes F \rightarrow \operatorname{R} \mathscr{H} o m(G, F)
$$

is an isomorphism. 
Let us apply this result when $X$ is a complex manifold. For $G \in \mathrm{D}_{\mathbb{R}-\mathrm{c}}^{\mathrm{b}}\left(\mathbb{C}_{X}\right)$, set

$$
\mathscr{A}_{G}=\mathscr{O}_{X} \otimes G, \quad \mathscr{B}_{G}:=\mathrm{R} \mathscr{H} \text { om }\left(\mathrm{D}_{X}^{\prime} G, \mathscr{O}_{X}\right)
$$

Note that if $X$ is the complexification of a real analytic manifold $M$ and we choose $G=\mathbb{C}_{M}$, we recover the sheaf of real analytic functions and the sheaf of hyperunctions:

$$
\mathscr{A}_{\mathbb{C}_{M}}=\mathscr{A}_{M}, \quad \mathscr{B}_{\mathbb{C}_{M}}=\mathscr{B}_{M}
$$

Now let $\mathscr{M} \in \mathrm{D}_{\text {coh }}^{\mathrm{b}}\left(\mathscr{D}_{X}\right)$. According to [ScSn94], one says that the pair $(G, \mathscr{M})$ is elliptic if $\operatorname{char}(\mathscr{M}) \cap \mathrm{SS}(G) \subset T_{X}^{*} X$.

Corollary 1.5.2. ScSn94 Let $(\mathscr{M}, G)$ be an elliptic pair.

(a) We have the canonical isomorphism:

$$
\mathrm{R} \mathscr{H}_{0 m_{X}}\left(\mathscr{M}, \mathscr{A}_{G}\right) \stackrel{\sim}{\sim} \mathscr{H}_{o m_{\mathscr{D}_{X}}}\left(\mathscr{M}, \mathscr{B}_{G}\right)
$$

(b) Assume moreover that $\operatorname{Supp}(\mathscr{M}) \cap \operatorname{Supp}(G)$ is compact. Then the cohomology of the complex $\mathrm{RHom}_{\mathscr{D}_{X}}\left(\mathscr{M}, \mathscr{A}_{G}\right)$ is finite dimensional.

To prove the part (b) of the corollary, one represents the left hand side of the global sections of (1.5.1) by a complex of topological vector spaces of type DFN and the right hand side by a complex of topological vector spaces of type FN. 


\title{
Lecture 2
}

\section{Microlocal Euler classes and Hochschild homology}

\begin{abstract}
This is a joint work with Masaki Kashiwara announced in [KS12]. On a complex manifold $\left(X, \mathscr{O}_{X}\right)$, the Hochschild homology is a powerful tool to construct characteristic classes of coherent modules and to get index theorems. Here, I will show how to adapt this formalism to a wide class of sheaves on a real manifold $M$ by using the functor $\mu$ hom of microlocalization. This construction applies in particular to constructible sheaves on real manifolds and $\mathscr{D}$-modules on complex manifolds, or more generally to elliptic pairs.
\end{abstract}

\subsection{Hochschild homology on complex mani- folds}

Hochschild homology of $\mathscr{O}$-modules has given rise to a vast literature. Let us quote in particular $\mathrm{Hu} 06, \mathrm{Ca05}, \mathrm{Ca07}, \mathrm{Ra08}$.

Consider a complex manifold $\left(X, \mathscr{O}_{X}\right)$ and denote by $\omega_{X}^{\text {hol }}$ the dualizing complex in the category of $\mathscr{O}_{X}$-modules, that is, $\omega_{X}^{\text {hol }}=\Omega_{X}\left[d_{X}\right]$, where $d_{X}$ is the complex dimension of $X$ and $\Omega_{X}$ is the sheaf of holomorphic forms of degree $d_{X}$. We shall use the classical six operations for $\mathscr{O}$-modules, $f^{*}, \mathrm{R} f_{*}$, $f^{!}, \mathrm{R} f_{!}, \stackrel{\mathrm{L}}{\otimes_{\mathscr{O}}}$ and $\mathrm{R} \mathscr{H} o m_{\mathscr{O}}$. In particular we have the two duality functors

$$
\begin{aligned}
& \mathrm{D}_{\mathscr{O}}^{\prime}(\bullet)=\operatorname{R} \mathscr{H}_{o m_{O_{X}}}\left(\cdot, \mathscr{O}_{X}\right) \\
& \mathrm{D}_{\mathscr{O}}(\bullet)=\operatorname{R} \mathscr{H}_{o m_{O_{X}}}\left(\cdot, \omega_{X}^{\text {hol }}\right)
\end{aligned}
$$

as well as the external product that we denote by $\stackrel{\mathrm{L}}{\mathscr{O}}_{\mathscr{O}}$. Denote by $\delta: X \hookrightarrow$ 
$X \times X$ the diagonal embedding and let $\Delta=\delta(X)$. We set

$$
\mathscr{O}_{\Delta}:=\delta_{*} \mathscr{O}_{X}, \quad \omega_{X}^{\text {hol, } \otimes-1}:=D_{\mathscr{O}}^{\prime} \omega_{X}^{\text {hol }}, \quad \omega_{\Delta}^{\text {hol, } \otimes-1}:=\delta_{*} \omega_{X}^{\text {hol, } \otimes-1}
$$

It is well-known that

$$
\omega_{\Delta}^{\text {hol, } \otimes-1} \simeq \mathrm{R} \mathscr{H} o m_{\mathscr{O}_{X \times X}}\left(\mathscr{O}_{\Delta}, \mathscr{O}_{X \times X}\right) .
$$

The Hochschild homology of $\mathscr{O}_{X}$ is usually defined by

$$
\mathscr{H} \mathscr{H}\left(\mathscr{O}_{X}\right)=\delta^{-1}\left(\mathscr{O}_{\Delta} \stackrel{\mathrm{L}}{\otimes}_{\mathscr{O}_{X \times X}} \mathscr{O}_{\Delta}\right) .
$$

Note the isomorphisms

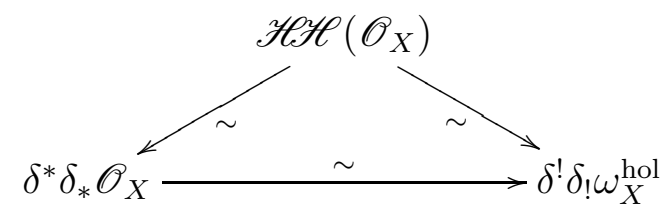

and the canonical isomorphisms

$$
\begin{aligned}
\delta^{*} \delta_{*} \mathscr{O}_{X} & \simeq \delta^{-1} \mathrm{R} \mathscr{H}_{o m_{\mathscr{O}_{X \times X}}}\left(\omega_{\Delta}^{\text {hol }, \otimes-1}, \mathscr{O}_{\Delta}\right), \\
\delta^{!} \delta_{!} \omega_{X}^{\text {hol }} & \simeq \delta^{-1} \mathrm{R} \mathscr{H}_{o m_{\mathscr{O}_{X \times X}}}\left(\mathscr{O}_{\Delta}, \omega_{\Delta}^{\text {hol }}\right) .
\end{aligned}
$$

For a closed subset $S$ of $X$, we set:

$$
\mathbb{H H}_{S}^{0}\left(\mathscr{O}_{X}\right)=H^{0}\left(X ; \mathrm{R} \Gamma_{S} \mathscr{H} \mathscr{H}\left(\mathscr{O}_{X}\right)\right)
$$

Let $\mathscr{F} \in \mathrm{D}_{\mathrm{coh}}^{\mathrm{b}}\left(\mathscr{O}_{X}\right)$. The morphisms $\mathrm{D}_{\mathscr{O}}^{\prime} \mathscr{F} \stackrel{\mathrm{L}}{\otimes_{\mathscr{O}_{X}}} \mathscr{F} \rightarrow \mathscr{O}_{X}$ and $\mathrm{D}_{\mathscr{O}} \mathscr{F}^{\mathrm{L}} \stackrel{\mathrm{Q}}{X}_{X}$ $\mathscr{F} \rightarrow \omega_{X}^{\text {hol }}$ give by adjunction the morphisms

$$
\mathrm{D}_{\mathscr{O}}^{\prime} \mathscr{F} \mathrm{L}_{\mathscr{O}_{X \times X}} \mathscr{F} \rightarrow \mathscr{O}_{\Delta}, \quad \mathrm{D}_{\mathscr{O}} \mathscr{F} \bigotimes_{\mathscr{O}_{X \times X}}^{\mathrm{L}} \mathscr{F} \rightarrow \omega_{\Delta}^{\text {hol }}
$$

and then by duality the morphisms

$$
\omega_{\Delta}^{\mathrm{hol}, \otimes-1} \rightarrow \mathrm{D}_{\mathscr{O}}^{\prime} \mathscr{F} \bigotimes_{\mathscr{O}_{X \times X}}^{\mathrm{L}} \mathscr{F} \rightarrow \mathscr{O}_{\Delta}, \quad \mathscr{O}_{\Delta} \rightarrow \mathrm{D}_{\mathscr{O}} \mathscr{F} \bigotimes_{\mathscr{O}_{X \times X}}^{\mathrm{L}} \mathscr{F} \rightarrow \omega_{\Delta}^{\mathrm{hol}}
$$

and the composition defines the Hochschild classes of $\mathscr{F}$ :

$(2.1 .5) \operatorname{hh}_{\mathscr{O}}(\mathscr{F}) \in H_{\operatorname{supp}(\mathscr{F})}^{0}\left(X ; \delta^{-1} \delta_{*} \mathscr{O}_{X}\right), \quad \widetilde{\operatorname{hh}}_{\mathscr{O}}(\mathscr{F}) \in H_{\operatorname{supp}(\mathscr{F})}^{0}\left(X ; \delta^{!} \delta_{!} \omega_{X}\right)$.

One can compose Hochschild homology and the Hochschild class commutes with the composition of kernels. More precisely, consider complex manifolds $X_{i}(i=1,2,3)$. 
- We write $X_{i j}:=X_{i} \times X_{j}(1 \leq i, j \leq 3), X_{123}=X_{1} \times X_{2} \times X_{3}, X_{1223}=$ $X_{1} \times X_{2} \times X_{2} \times X_{3}$, etc.

- We denote by $q_{i}$ the projection $X_{i j} \rightarrow X_{i}$ or the projection $X_{123} \rightarrow X_{i}$ and by $q_{i j}$ the projection $X_{123} \rightarrow X_{i j}$.

Let $K_{i j} \in \mathrm{D}_{\text {coh }}^{\mathrm{b}}\left(\mathscr{O}_{X_{i j}}\right)(i=1,2, j=i+1)$. One sets

$$
K_{12}{ }_{2}^{\circ} K_{23}=\mathrm{R} q_{13 !}\left(q_{12}^{*} K_{12} \stackrel{\mathrm{L}}{\otimes}_{\mathscr{O}_{X_{123}}} q_{23}^{*} K_{23}\right)
$$

Theorem 2.1.1. (a) There is a natural morphism

$$
\mathscr{H} \mathscr{H}\left(\mathscr{O}_{X_{12}}\right) \underset{2}{\circ} \mathscr{H} \mathscr{H}\left(\mathscr{O}_{X_{23}}\right) \rightarrow \mathscr{H} \mathscr{H}\left(\mathscr{O}_{X_{13}}\right)
$$

(b) Let $S_{i j} \subset X_{i j}$ be a closed subset $(i=1,2, j=i+1)$. Assume that $q_{13}$ is proper over $S_{12} \times_{X_{2}} S_{23}$ and set $S_{13}=q_{13}\left(S_{12} \times_{X_{2}} S_{23}\right)$. Then the morphism above induces a map

$$
\stackrel{\circ}{2}: \mathbb{H}_{S_{12}}^{0}\left(\mathscr{O}_{X_{12}}\right) \otimes \mathbb{H}_{S_{23}}^{0}\left(\mathscr{O}_{X_{23}}\right) \rightarrow \mathbb{H}_{S_{13}}^{0}\left(\mathscr{O}_{X_{13}}\right)
$$

(c) Let $K_{i j}$ be as above and assume that $\operatorname{supp}\left(K_{i j}\right) \subset S_{i j}$. Set $K_{13}=$ $K_{12} \circ K_{23}$ and $\widetilde{K}_{13}=\left(K_{12} \otimes \omega_{2}^{\text {hol } \otimes-1}\right)_{2}^{\circ} K_{23}$. Then $K_{13}$ and $\widetilde{K}_{13}$ belong to $\mathrm{D}_{\text {coh }}^{\mathrm{b}}\left(\mathscr{O}_{X_{13}}\right)$ and we have the equalities in $\mathbb{H}_{H_{S_{13}}^{0}}^{0}\left(\mathscr{O}_{X_{13}}\right)$ :

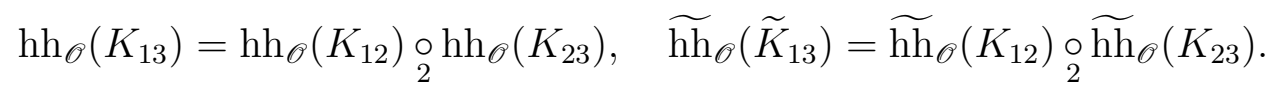

This theorem shows in particular that the Hochschild class commutes with external product, inverse image and proper direct image.

Theorem 2.1.1 seems to be well-known from the specialists although it is difficult to find a precise statement (see however [Ca07, Ra10]). The construction of the Hochschild homology as well as Theorem 2.1.1 (including complete proofs) have been extended when replacing $\mathscr{O}_{X}$ with a so-called DQ-algebroid stack $\mathscr{A}_{X}$ in $\mathrm{KS} 12 \mathrm{~b}$.

Coming back to $\mathscr{O}_{X}$-modules, the Hodge cohomology of $\mathscr{O}_{X}$ is given by:

$$
\mathcal{H D}\left(\mathscr{O}_{X}\right):=\bigoplus_{i=0}^{d_{X}} \Omega_{X}^{i}[i] \text {, an object of } \mathrm{D}^{\mathrm{b}}\left(\mathscr{O}_{X}\right)
$$

There is a commutative diagram constructed by Kashiwara in Ka91 in which $\alpha_{X}$ is the HKR (Hochschild-Kostant-Rosenberg) isomorphism and $\beta_{X}$ is a 
kind of dual HKR isomorphism:

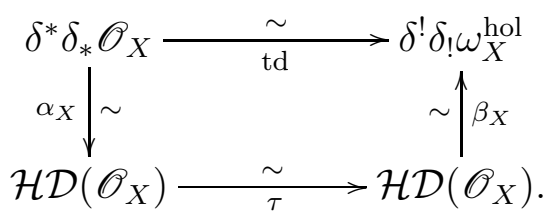

If $\mathscr{F} \in \mathrm{D}_{\mathrm{coh}}^{\mathrm{b}}\left(\mathscr{O}_{X}\right)$, the Chern character of $\mathscr{F}$ is the image by $\alpha_{X}$ of $\mathrm{hh}_{\mathscr{O}}(\mathscr{F})$.

In Ka91 Kashiwara made the conjecture that the arrow $\tau$ making the diagram commutative is given by the cup product by the Todd class of $X$. This conjecture has recently been proved by Ramadoss [Ra08] in the algebraic case (after preliminary important results by Markarian) and Grivaux Gr09] in the analytic case (and with a very simple proof). Since the morphism $\beta_{X}$ commutes with proper direct images, we get a new and functorial approach to the Riemann-Roch-Hirzebruch-Grothendieck theorem.

\subsection{Microlocal homology}

We keep the notations of Lecture I. In particular $\omega_{M}$ denotes the dualizing complex on $M$ and $\mathrm{D}_{M}^{\prime}$ is the duality functor. We set

$$
\omega_{\Delta}:=\delta_{*} \omega_{M}, \quad \omega_{M}^{\otimes-1}:=\mathrm{D}_{M}^{\prime} \omega_{M}, \quad \omega_{\Delta}^{\otimes-1}:=\delta_{*} \omega_{M}^{\otimes-1} .
$$

Let $M_{i}(i=1,2,3)$ be manifolds.

- For short, we write as above $M_{i j}:=M_{i} \times M_{j}(1 \leq i, j \leq 3), M_{123}=$ $M_{1} \times M_{2} \times M_{3}$, etc.

- We will often write for short $\mathbf{k}_{i}$ instead of $\mathbf{k}_{M_{i}}$ and $\mathbf{k}_{\Delta_{i}}$ instead of $\mathbf{k}_{\Delta_{M_{i}}}$, $\pi_{i}$ instead of $\pi_{M_{i}}$, etc.

- We denote by $q_{i}$ the projection $M_{i j} \rightarrow M_{i}$ or the projection $M_{123} \rightarrow M_{i}$ and by $q_{i j}$ the projection $M_{123} \rightarrow M_{i j}$. Similarly, we denote by $p_{i}$ the projection $T^{*} M_{i j} \rightarrow T^{*} M_{i}$ or the projection $T^{*} M_{123} \rightarrow T^{*} M_{i}$ and by $p_{i j}$ the projection $T^{*} M_{123} \rightarrow T^{*} M_{i j}$.

- We also need tointroduce the maps $p_{j^{a}}$ or $p_{i j^{a}}$, the composition of $p_{j}$ or $p_{i j}$ and the antipodal map on $T^{*} M_{j}$.

We consider the operations of composition of kernels. For $K_{i j} \in \mathrm{D}^{\mathrm{b}}\left(\mathbf{k}_{M_{i j}}\right)$ $(i=1,2, j=i+1)$, we set

$$
\begin{aligned}
& K_{1} \circ K_{2}:=\operatorname{R} q_{13 !} \delta_{2}^{-1}\left(K_{1} \otimes K_{2}\right) \simeq \mathrm{R} q_{13 !}\left(q_{12}^{-1} K_{1} \otimes q_{23}^{-1}\right), \\
& K_{1} \underset{2}{*} K_{2}:=\operatorname{R} q_{13 *}\left(\delta_{2}^{!}\left(K_{1} \otimes K_{2}\right) \otimes q_{2}^{-1} \omega_{2}\right) .
\end{aligned}
$$


We have a natural morphism $K_{1} \circ K_{2} \rightarrow K_{1} * K_{2}$. It is an isomorphism if $p_{12^{a}}^{-1} \mathrm{SS}\left(K_{1}\right) \cap p_{23^{a}}^{-1} \mathrm{SS}\left(K_{2}\right) \rightarrow T^{*} M_{13}$ is proper.

We also define the composition of kernels on cotangent bundles. For $L_{i} \in \mathrm{D}^{\mathrm{b}}\left(\mathbf{k}_{T_{M_{i j}}^{*}}\right)(i=1,2, j=i+1)$, we set

$$
L_{1} \stackrel{a}{\circ} L_{2}:=\operatorname{R} p_{13^{a} !}\left(p_{12^{a}}^{-1} L_{1} \otimes p_{23^{a}}^{-1} L_{2}\right) .
$$

For $K_{1}, F_{1} \in \mathrm{D}^{\mathrm{b}}\left(\mathbf{k}_{M_{12}}\right)$ and $K_{2}, F_{2} \in \mathrm{D}^{\mathrm{b}}\left(\mathbf{k}_{M_{23}}\right)$ there exists a canonical morphism:

$$
\mu h o m\left(K_{1}, F_{1}\right) \underset{2}{\stackrel{a}{\circ}} \mu h o m\left(K_{2}, F_{2}\right) \rightarrow \mu h o m\left(K_{1} \underset{2}{*} K_{2}, F_{1} \underset{2}{\circ} F_{2}\right) .
$$

We also define the corresponding operations for subsets of cotangent bundles. Let $A \subset T^{*} M_{12}$ and $B \subset T^{*} M_{23}$. We set $\underset{2}{\stackrel{a}{\stackrel{a}{a}} B} B=p_{13}(A \underset{2}{\stackrel{a}{\times} B})$ where $A \stackrel{a}{\times} B=p_{12^{a}}^{-1}(A) \cap p_{23}^{-1}(B)$.

If there is no risk of confusion, we simply denote by $\delta^{a}$ the map:

$$
\delta^{a}: T^{*} M \hookrightarrow T^{*}(M \times M), \quad(x ; \xi) \mapsto(x, x ; \xi,-\xi) .
$$

Definition 2.2.1. Let $\Lambda$ be a closed conic subset of $T^{*} M$. We set

$$
\begin{aligned}
\mathscr{M} \mathscr{H}\left(\mathbf{k}_{M}\right) & :=\left(\delta^{a}\right)^{-1} \mu h o m\left(\mathbf{k}_{\Delta_{M}}, \omega_{\Delta_{M}}\right), \\
\mathbb{M H}_{\Lambda}^{0}\left(\mathbf{k}_{M}\right) & :=H_{\Lambda}^{0}\left(T^{*} M ; \mathscr{M} \mathscr{H}\left(\mathbf{k}_{M}\right)\right) .
\end{aligned}
$$

We call $\mathscr{M} \mathscr{H}\left(\mathbf{k}_{M}\right)$ the microlocal homology of $M$.

We have isomorphisms

$$
\mathscr{M} \mathscr{H}\left(\mathbf{k}_{M}\right) \simeq\left(\delta^{a}\right)^{-1} \mu_{\Delta}\left(\omega_{\Delta}\right) \simeq \pi_{M}^{-1} \omega_{M}
$$

and the isomorphism $\mathscr{M} \mathscr{H}\left(\mathbf{k}_{M}\right) \simeq \pi_{M}^{-1} \omega_{M}$ plays the role of the HKR isomorphism in the complex case.

We have the analogue of Theorem 2.1.1 (a) and (b). (For the part (c), see Theorem 2.3.1 below.)

Let $i=1,2, j=i+1$ and let $\Lambda_{i j}$ be a closed conic subset of $T^{*} M_{i j}$. Assume that

$$
\Lambda_{12} \underset{2}{\stackrel{a}{\times}} \Lambda_{23} \text { is proper over } T^{*} M_{13} .
$$

Note that this hypothesis is equivalent to

$$
\left\{\begin{array}{l}
p_{12^{a}}^{-1}\left(\Lambda_{12}\right) \cap p_{23^{a}}^{-1}\left(\Lambda_{23}\right) \cap\left(T_{M_{1}}^{*} M_{1} \times T^{*} M_{2} \times T_{M_{3}}^{*} M_{3}\right) \subset T_{M_{123}}^{*} M_{123} \\
q_{13} \text { is proper on } \pi_{12}\left(\Lambda_{12}\right) \times_{M_{2}} \pi_{23}\left(\Lambda_{23}\right)
\end{array}\right.
$$


Set

$$
\Lambda_{13}=\Lambda_{12} \underset{2}{\stackrel{a}{\circ}} \Lambda_{23}
$$

Theorem 2.2.2. (a) There is a natural morphism

$$
\mathscr{M} \mathscr{H}\left(\mathbf{k}_{M_{12}}\right)_{2}^{\circ} \mathscr{M} \mathscr{H}\left(\mathbf{k}_{M_{23}}\right) \rightarrow \mathscr{M} \mathscr{H}\left(\mathbf{k}_{M_{13}}\right)
$$

(b) Let $\Lambda_{i j} \subset T^{*} M_{i j}$ be as above and assume (2.2.3). Then morphism (2.2.5) induces a map

$$
\stackrel{\circ}{2}: \mathbb{M H}_{\Lambda_{12}}^{0}\left(\mathbf{k}_{M_{12}}\right) \otimes \mathbb{M H}_{\Lambda_{23}}^{0}\left(\mathbf{k}_{X_{23}}\right) \rightarrow \mathbb{M H}_{\Lambda_{13}}^{0}\left(\mathbf{k}_{X_{13}}\right)
$$

The construction of the morphism (2.2.5) uses (2.2.2), which makes the computations not easy. Fortunately, we have the following result.

Proposition 2.2.3. Let $M_{i}(i=1,2,3)$ be manifolds and let $\Lambda_{i j}$ be a closed conic subset of $T^{*} M_{i j}(i j=12,13,23)$. We have a commutative diagram

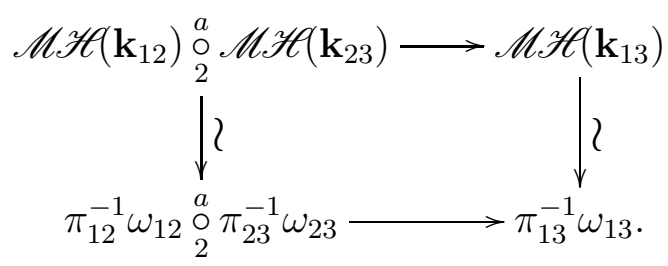

Here the bottom horizontal arrow is induced by

$$
\begin{aligned}
& p_{12^{a}}^{-1} \pi_{12}^{-1} \omega_{12} \otimes p_{23^{a}}^{-1} \pi_{23^{a}}^{-1} \omega_{23} \simeq \pi_{1}^{-1} \omega_{1} \bigotimes \omega_{T^{*} M_{2}} \otimes \pi_{3}^{-1} \omega_{3} \\
& \text { and } \\
& \operatorname{R} p_{13^{a} !}\left(\pi_{1}^{-1} \omega_{1} \bigotimes \omega_{T^{*} M_{2}} \otimes \pi_{M_{3}}^{-1} \omega_{3}\right) \longrightarrow \pi_{1}^{-1} \omega_{1} \bigotimes \pi_{3}^{-1} \omega_{3} .
\end{aligned}
$$

Remark 2.2.4. (i) If we consider that the isomorphism $\mathscr{M} \mathscr{H}\left(\mathbf{k}_{M}\right) \simeq \pi^{-1} \omega_{M}$ is a real analogue of the Hochschild-Kostant-Rosenberg isomorphism, then the commutativity of Diagram (2.2.7) says that, contrarily to the complex case, the real HKR isomorphism commutes with inverse and direct images.

(ii) As a particular case of Proposition 2.2.3, we get canonical isomorphisms

$$
\mathscr{M} \mathscr{H}\left(\mathbf{k}_{M}\right) \otimes \mathscr{M} \mathscr{H}\left(\mathbf{k}_{M}\right) \simeq \pi^{-1} \omega_{M} \otimes \pi^{-1} \omega_{M} \simeq \omega_{T^{*} M}
$$

Hence, $\mathscr{M} \mathscr{H}\left(\mathbf{k}_{M}\right)$ behaves as a "square root" of the dualizing complex. 


\subsection{Trace kernels and microlocal Euler classes}

A trace kernel $(K, u, v)$ on $M$ is the data of $K \in \mathrm{D}^{\mathrm{b}}\left(\mathbf{k}_{M \times M}\right)$ together with morphisms $(u, v)$

$$
\mathbf{k}_{\Delta} \stackrel{u}{\rightarrow} K \stackrel{v}{\rightarrow} \omega_{\Delta}
$$

Setting $\mathrm{SS}_{\Delta}(K):=\mathrm{SS}(K) \cap T_{\Delta}^{*}(M \times M)$, the morphism $u$ gives an element of $H_{\mathrm{SS}_{\Delta}(K)}^{0}\left(T^{*} M ; \mu h o m\left(\mathbf{k}_{\Delta}, K\right)\right)$ whose image by $v$ is the microlocal Euler class of $K$

$$
\left.\mu \mathrm{eu}_{M}(K) \in \mathbb{M H}_{\mathrm{SS}_{\Delta}(K)}^{0}\left(\mathbf{k}_{M}\right)\right) \simeq H_{\mathrm{SS}_{\Delta}(K)}^{0}\left(T^{*} M ; \pi^{-1} \omega_{M}\right)
$$

If $M=$ pt, a Hochschild kernel $K$ is nothing but an object of $\mathrm{D}^{\mathrm{b}}(\mathbf{k})$ together with linear maps $\mathbf{k} \rightarrow K \rightarrow \mathbf{k}$. The composition gives the element $\mu \mathrm{eu}(K)$ of $\mathbf{k}$. If $\mathbf{k}$ is a field of characteristic zero and $K=L \otimes L^{*}$ where $L$ is a bounded complex of $\mathbf{k}$-modules with finite dimensional cohomology and $L^{*}$ is its dual, one recovers the classical Euler-Poincaré index of $L$, that is, $\mu \mathrm{eu}(K)=\chi(L)$.

Let $i=1,2, j=i+1$ and let $\Lambda_{i i j j}$ be a closed conic subset of $T^{*} M_{i i j j}$. Assume that

$$
\Lambda_{1122} \underset{22}{\stackrel{a}{\times}} \Lambda_{2233} \text { is proper over } T^{*} M_{1133} .
$$

Set $\Lambda_{1133}=\Lambda_{1122} \underset{22}{\stackrel{a}{\circ}} \Lambda_{2233}$ and $\Lambda_{i j}=\Lambda_{i i j j} \cap T_{\Delta_{i j}}^{*} M_{i i j j}$.

Theorem 2.3.1. Let $K_{i j}$ be a trace kernel on $M_{i j}$ with $\operatorname{SS}\left(K_{i j}\right) \subset \Lambda_{i i j j}$. Assume (2.3.1), set $\widetilde{K}_{23}=\omega_{\Delta_{2}}^{\otimes-1}{ }_{2} K_{23} \simeq\left(\omega_{2}^{\otimes-1} \otimes \mathbf{k}_{233}\right) \stackrel{\mathrm{L}}{\otimes} K_{23}$ and set $K_{13}=$ $K_{12} \underset{22}{\circ} \widetilde{K}_{23}$. Then

(a) $K_{13}$ is a trace kernel on $M_{13}$,

(b) $\mu \mathrm{eu}_{M_{13}}\left(K_{13}\right)=\mu \mathrm{eu}_{M_{12}}\left(K_{12}\right) \underset{2}{\stackrel{a}{\circ}} \mu \mathrm{eu}_{M_{23}}\left(K_{23}\right)$ as elements of $\mathbb{M H}_{\Lambda_{13}}^{0}\left(\mathbf{k}_{13}\right)$.

As an application, one can perform the external product, the proper direct image and the non characteristic inverse image of trace kernels and compute their microlocal Euler classes.

Consider in particular the case where $\Lambda_{1}$ and $\Lambda_{2}$ are two closed conic subsets of $T^{*} M$ satisfying the transversality condition

$$
\Lambda_{1} \cap \Lambda_{2}^{a} \subset T_{M}^{*} M
$$


Then applying Theorem 2.3.1 and composing the external product with the restriction to the diagonal, we get a convolution map:

$$
\star: \mathbb{M H}_{\Lambda_{1}}\left(\mathbf{k}_{M}\right) \times \mathbb{M H}_{\Lambda_{2}}\left(\mathbf{k}_{M}\right) \rightarrow \mathbb{M H}_{\Lambda_{1}+\Lambda_{2}}\left(\mathbf{k}_{M}\right) .
$$

Proposition 2.3.2. Let $K_{i}$ be a trace kernels with $\mathrm{SS}_{\Delta}\left(K_{i}\right) \subset \Lambda_{i}(i=1,2)$ and assume (2.3.1). Then the object $K_{1} \stackrel{\mathrm{L}}{\otimes}\left(\mathbf{k}_{M} \stackrel{\mathrm{L}}{\otimes} \omega_{M}^{\otimes-1}\right) \stackrel{\mathrm{L}}{\otimes} K_{2}$ is a trace kernel on $M$ and

$$
\mu \mathrm{eu}_{M}\left(K_{1} \stackrel{\mathrm{L}}{\otimes}\left(\mathbf{k}_{M} \stackrel{\mathrm{L}}{\otimes} \omega_{M}^{\otimes-1}\right) \stackrel{\mathrm{L}}{\otimes} K_{2}\right)=\mu \mathrm{eu}_{M}\left(K_{1}\right) \star \mu \mathrm{eu}_{M}\left(K_{2}\right) .
$$

In particular if $\operatorname{supp} K_{1} \cap \operatorname{supp} K_{2}$ is compact, we have

$$
\begin{aligned}
\mu \mathrm{eu}\left(\mathrm{R} \Gamma\left(M \times M ; K_{1} \stackrel{\mathrm{L}}{\otimes}\left(\mathbf{k}_{M} \stackrel{\mathrm{L}}{\otimes} \omega_{M}^{\otimes-1}\right) \stackrel{\mathrm{L}}{\otimes} K_{2}\right)\right) & =\left.\int_{M}\left(\mu \mathrm{eu}\left(K_{1}\right) \star \mu \mathrm{eu}\left(K_{2}\right)\right)\right|_{M} \\
& =\int_{T^{*} M} \mu \mathrm{eu}\left(K_{1}\right) \cup \mu \mathrm{eu}\left(K_{2}\right) .
\end{aligned}
$$

We shall apply this result to elliptic pairs.

\subsection{Microlocal Euler class of constructible sheaves}

Let us denote by $\mathrm{D}_{\mathrm{cc}}^{\mathrm{b}}\left(\mathbf{k}_{M}\right)$ the full triangulated subcategory of $\mathrm{D}^{\mathrm{b}}\left(\mathbf{k}_{M}\right)$ consisting of cohomologically constructible sheaves and let $G \in \mathrm{D}_{\mathrm{cc}}^{\mathrm{b}}\left(\mathbf{k}_{M}\right)$.

The evaluation morphism $G \stackrel{\mathrm{L}}{\otimes} \mathrm{D}_{M} G \rightarrow \omega_{M}$ gives by adjunction the morphism $G \stackrel{\mathrm{L}}{\otimes} \mathrm{D}_{M} G \rightarrow \omega_{\Delta}$. By duality, one gets the morphism $\mathbf{k}_{\Delta} \rightarrow G \stackrel{\mathrm{L}}{\square} \mathrm{D}_{M} G$. To summarize, we have the morphisms in $\mathrm{D}_{\mathrm{cc}}^{\mathrm{b}}\left(\mathbf{k}_{M \times M}\right)$ :

$$
\mathbf{k}_{\Delta} \rightarrow G \stackrel{\mathrm{L}}{\otimes} \mathrm{D} G \rightarrow \omega_{\Delta} .
$$

Denote by $\operatorname{TK}(G)$ the Hochschild kernel so constructed. If $G$ is $\mathbb{R}$-constructible, the class $\mu \mathrm{eu}_{M}(\mathrm{TK}(G))$ is nothing but the Lagrangian cycle of $G$ constructed by Kashiwara [Ka85]. In the sequel, if there is no risk of confusion, we simply denote this class by $\mu \mathrm{eu}_{M}(G)$.

One recovers the classical functorial properties of Lagrangian cycles. Let $f: M \rightarrow N$ be a morphism of manifolds. To $f$ one associates the maps

$$
T^{*} M \stackrel{f_{d}}{\longleftarrow} M \times_{N} T^{*} N \stackrel{f_{\pi}}{\longrightarrow} T^{*} N
$$

There are natural morphsim

$$
\begin{aligned}
& f_{\mu}: f_{\pi !} f_{d}^{-1} \pi_{M}^{-1} \omega_{M} \rightarrow \pi_{N}^{-1} \omega_{N}, \\
& f^{\mu}: f_{d !} f_{\pi}^{-1} \pi_{N}^{-1} \omega_{N} \rightarrow \pi_{M}^{-1} \omega_{M} .
\end{aligned}
$$


- Let $F \in \mathrm{D}_{\mathbb{R}-\mathrm{c}}^{\mathrm{b}}\left(\mathbf{k}_{M}\right)$ and assume $f$ is proper on $\operatorname{supp}(F)$, or equivalently, $f_{\pi}$ is proper on $f_{d}^{-1} \mathrm{SS}(F)$. Then $\mu \mathrm{eu}\left(\mathrm{R} f_{*} F\right)=f_{\mu} \mu \mathrm{eu}(F)$,

- Let $G \in \mathrm{D}_{\mathbb{R}-\mathrm{c}}^{\mathrm{b}}\left(\mathbf{k}_{N}\right)$ and assume that $f$ is non characteristic for $G$, that is, $f_{d}$ is proper on $f_{\pi}^{-1} \mathrm{SS}(G)$. Then $\mu \mathrm{eu}\left(f^{-1} G\right)=f^{\mu} \mu \mathrm{eu}(D)$.

\subsection{Microlocal Euler class of $\mathscr{D}$-modules}

In this section, we denote by $X$ a complex manifold of complex dimension $d_{X}$ and the base ring $\mathbf{k}$ is the field $\mathbb{C}$. One denotes by $\mathscr{D}_{X}$ the sheaf of $\mathbb{C}_{X^{-}}$ algebras of (finite order) holomorphic differential operators on $X$ and refer to [Ka03] for a detailed exposition of the theory of $\mathscr{D}$-modules.

We also denote by $\mathrm{D}_{\text {coh }}^{\mathrm{b}}\left(\mathscr{D}_{X}\right)$ the full triangulated subcategory of $\mathrm{D}^{\mathrm{b}}\left(\mathscr{D}_{X}\right)$ consisting of objects with coherent cohomology. We denote by $\mathrm{D}_{\mathscr{D}}: \mathrm{D}^{\mathrm{b}}\left(\mathscr{D}_{X}\right) \rightarrow$ $\mathrm{D}^{\mathrm{b}}\left(\mathscr{D}_{X}\right)$ the duality functor for left $\mathscr{D}$-modules:

$$
\mathrm{D}_{\mathscr{D}} \mathscr{M}:=\mathrm{R} \mathscr{H} O m_{\mathscr{D}_{X}}\left(\mathscr{M}, \mathscr{D}_{X}\right) \otimes_{\mathscr{O}_{X}} \omega_{X}^{\text {hol, }, \otimes-1}
$$

We denote by $\bullet \underline{\otimes} \cdot$ the external product for $\mathscr{D}$-modules:

$$
\mathscr{M} \underline{\otimes} \mathscr{N}:=\mathscr{D}_{X \times X} \otimes_{\mathscr{D}_{X} \otimes \mathscr{D}_{X}}(\mathscr{M} \otimes \mathscr{N}) .
$$

Let $\Delta$ be the diagonal of $X \times X$. The left $\mathscr{D}_{X \times X}$-module $H_{[\Delta]}^{d_{X}}\left(\mathscr{O}_{X \times X}\right)$ (the algebraic cohomology with support in $\Delta$ ) is denoted as usual by $\mathscr{B}_{\Delta}$. We also introduce $\mathscr{B}_{\Delta}^{\vee}:=\mathscr{B}_{\Delta}\left[2 d_{X}\right]$. For a coherent $\mathscr{D}_{X}$-module $\mathscr{M}$, we have the isomorphism

$$
\mathrm{R} \mathscr{H}_{o m_{\mathscr{D}_{X}}}(\mathscr{M}, \mathscr{M}) \simeq \mathrm{R} \mathscr{H} o m_{\mathscr{D}_{X \times X}}\left(\mathscr{B}_{\Delta}, \mathscr{M} \underline{\underline{\nabla}} \mathrm{D}_{\mathscr{D}} \mathscr{M}\right)\left[d_{X}\right] .
$$

We get the morphisms

$$
\mathscr{B}_{\Delta} \rightarrow \mathscr{M} \underline{\underline{\nabla}} \mathrm{D}_{\mathscr{D}} \mathscr{M}\left[d_{X}\right] \rightarrow \mathscr{B}_{\Delta}^{\vee}
$$

where the second morphism is deduced by duality.

Denote by $\mathscr{E}_{T^{*} X}$ the sheaf on $T^{*} X$ of microdifferential operators of [SKK73]. For a coherent $\mathscr{D}_{X}$-module $\mathscr{M}$ set

$$
\mathscr{M}^{E}:=\mathscr{E}_{T^{*} X} \otimes_{\pi^{-1} \mathscr{D}_{X}} \pi^{-1} \mathscr{M}
$$

Recall that, denoting by $\operatorname{char}(\mathscr{M})$ the characteristic variety of $\mathscr{M}$, we have $\operatorname{char}(\mathscr{M})=\operatorname{supp}\left(\mathscr{M}^{E}\right)$. Set

$$
\mathscr{C}_{\Delta}:=\mathscr{B}_{\Delta}^{E}, \quad \mathscr{C}_{\Delta}^{\vee}:=\left(\mathscr{B}_{\Delta}^{\vee}\right)^{E} .
$$


Let $\Lambda$ be a closed conic subset of $T^{*} X$. One sets

$$
\begin{aligned}
& \mathscr{H} \mathscr{H}\left(\mathscr{E}_{T^{*} X}\right)=\left(\delta^{a}\right)^{-1} \mathrm{R} \mathscr{H}_{o m_{\mathscr{E}_{X \times X}}}\left(\mathscr{C}_{\Delta}, \mathscr{C}_{\Delta}^{\vee}\right), \\
& \mathbb{H}_{H_{\Lambda}^{0}}^{0}\left(\mathscr{E}_{T^{*} X}\right)=H_{\Lambda}^{0}\left(T^{*} X ; \mathscr{H} \mathscr{H}\left(\mathscr{E}_{T^{*} X}\right)\right) .
\end{aligned}
$$

One calls $\mathscr{H} \mathscr{H}\left(\mathscr{E}_{T^{*} X}\right)$ the Hochschild homology of $\mathscr{E}_{T^{*} X}$.

We deduce from (2.5.1) the morphisms

$$
\mathscr{C}_{\Delta} \rightarrow\left(\mathscr{M} \underline{\underline{\nabla}} D_{\mathscr{D}} \mathscr{M}\right)^{E}\left[d_{X}\right] \rightarrow \mathscr{C}_{\Delta}^{\vee}
$$

which define the Hochschild class of $\mathscr{M}$ :

$$
\operatorname{hh}_{\mathscr{E}}(\mathscr{M}) \in \mathbb{H} \mathbb{H}_{\operatorname{char}(\mathscr{M})}^{0}\left(\mathscr{E}_{T^{*} X}\right) .
$$

We shall make a link between the Hochschild class of $\mathscr{M}$ and the microlocal Euler class of a Hochschild kernel attached to the sheaf of holomorphic solutions of $\mathscr{M}$. We have

$$
\begin{aligned}
& \Omega_{X \times X}\left[-d_{X}\right] \stackrel{\mathrm{L}}{\otimes_{\mathscr{D}_{X \times X}}} \mathscr{B}_{\Delta} \simeq \mathbb{C}_{\Delta}, \\
& \Omega_{X \times X}\left[-d_{X}\right] \stackrel{\mathrm{L}}{{ }_{\mathscr{D}_{X \times X}}} \mathscr{B}_{\Delta}^{\vee} \simeq \omega_{\Delta} .
\end{aligned}
$$

Now remark that for $\mathscr{N}_{1}, \mathscr{N}_{2} \in \mathrm{D}_{\text {coh }}^{\mathrm{b}}\left(\mathscr{D}_{X}\right)$, we have a natural morphism

$$
\operatorname{R} \mathscr{H}_{0 m_{\pi^{-1} \mathscr{D}_{X}}}\left(\pi^{-1} \mathscr{N}_{1}, \mathscr{N}_{2}^{E}\right) \rightarrow \mu \operatorname{hom}\left(\Omega_{X} \stackrel{\mathrm{L}}{\mathscr{D}_{X}} \mathscr{N}_{1}, \Omega_{X} \stackrel{\mathrm{L}}{\otimes}_{\mathscr{D}_{X}} \mathscr{N}_{2}\right) .
$$

One deduces the morphisms

$$
\begin{aligned}
\operatorname{RHom}_{\mathscr{E}_{X \times X}}\left(\mathscr{C}_{\Delta},\right. & \left.\mathscr{C}_{\Delta}^{\vee}\right) \simeq \operatorname{R} \mathscr{H}_{o m} \pi_{\pi^{-1} \mathscr{D}_{X \times X}}\left(\pi^{-1} \mathscr{B}_{\Delta},\left(\mathscr{B}_{\Delta}^{\vee}\right)^{E}\right) \\
& \rightarrow \mu h o m\left(\Omega_{X \times X}{\stackrel{\mathrm{L}}{\mathscr{D}_{X \times X}}}_{\mathscr{B}_{\Delta}}{ }^{\otimes-1}, \Omega_{X \times X} \stackrel{\mathrm{L}}{\mathscr{\otimes}_{\mathscr{D}_{X \times X}}} \mathscr{B}_{\Delta}\right) \\
& \simeq \mu h o m\left(\mathbb{C}_{\Delta}, \omega_{\Delta}\right) .
\end{aligned}
$$

Since all the arrows above are isomorphisms, we get

$$
\mathscr{H} \mathscr{H}\left(\mathscr{E}_{T^{*} X}\right) \simeq \mathscr{M} \mathscr{H}\left(\mathbb{C}_{X}\right) .
$$

Recall that the Hochschild homology of $\mathscr{E}_{T^{*} X}$ has been already calculated in BG87].

By this isomorphism, $\operatorname{hh}_{\mathscr{E}}(\mathscr{M})$ belongs to $\mathbb{M H}_{\text {char }(\mathscr{M})}^{0}\left(\mathbb{C}_{X}\right)$ and this class coincides with that already introduced in [ScSn94].

Applying the functor $\Omega_{X \times X}\left[-d_{X}\right]{\stackrel{\mathrm{L}}{\otimes_{\mathscr{D}_{X \times X}}}}$. to the morphisms in (2.5.1) we get the morphisms

$$
\mathbb{C}_{\Delta} \rightarrow \Omega_{X \times X} \stackrel{\mathrm{L}}{\otimes_{\mathscr{D} X \times X}}\left(\mathscr{M} \underline{\otimes} \mathrm{D}_{\mathscr{D}} \mathscr{M}\right) \rightarrow \omega_{\Delta}
$$


For $\mathscr{M} \in \mathrm{D}_{\text {coh }}^{\mathrm{b}}\left(\mathscr{D}_{X}\right)$, we set

$$
\operatorname{TK}(\mathscr{M}):=\Omega_{X \times X} \stackrel{\mathrm{L}}{\otimes}_{\mathscr{D}_{X \times X}}\left(\mathscr{M} \underline{\nabla}_{\mathscr{D}} \mathscr{M}\right) .
$$

Then $\operatorname{TK}(\mathscr{M})$ is a trace kernel by (2.5.4) and $\mu \mathrm{eu}_{M}(\mathrm{TK}(\mathscr{M}))$ is supported by $\operatorname{char}(\mathscr{M})$ by Theorem 1.3.4.

Proposition 2.5.1. The Hochschild class of $\mathscr{M}$ is the microlocal Euler class of the trace kernel associated to $\mathscr{M}$, that is, $\operatorname{hh}_{\mathscr{E}}(\mathscr{M})=\mu \mathrm{eu}_{X}(\operatorname{TK}(\mathscr{M}))$ in $H_{\text {char }(\mathscr{M})}^{0}\left(T^{*} X ; \pi^{-1} \omega_{X}\right)$.

\subsection{Microlocal Euler class of elliptic pairs}

Let $X$ be a complex manifold, $\mathscr{M}$ an object of $\mathrm{D}_{\text {coh }}^{\mathrm{b}}\left(\mathscr{D}_{X}\right)$ and $G$ an object of $\mathrm{D}_{\mathbb{R} \text {-c }}^{\mathrm{b}}\left(\mathbb{C}_{X}\right)$. The pair $(\mathscr{M}, G)$ is called an elliptic pair in [ScSn94] if $\operatorname{char}(\mathscr{M}) \cap$ $\operatorname{SS}(G) \subset T_{X}^{*} X$. From now on, we assume that $(\mathscr{M}, G)$ is an elliptic pair. We set

$$
\operatorname{TK}(\mathscr{M}, G):=\Omega_{X \times X} \stackrel{\mathrm{L}}{\otimes}_{\mathscr{D} X \times X}\left((\mathscr{M} \otimes G) \underline{\otimes}\left(\mathrm{D}_{\mathscr{D}} \mathscr{M} \otimes \mathrm{D}_{X}^{\prime} G\right)\right) .
$$

It follows from the preceding results that $\operatorname{TK}(\mathscr{M}, G)$ is a trace kernel and

$$
\mu \mathrm{eu}_{X}(\operatorname{TK}(\mathscr{M}, G))=\mu \mathrm{eu}_{X}(\mathscr{M}) \star \mu \mathrm{eu}_{X}(G) .
$$

Applying Corollary 1.5.2 (a), we get the natural isomorphism

$$
\mathrm{R} \mathscr{H} o m_{\mathscr{D}_{X}}\left(\mathscr{M}, \mathrm{D}_{X}^{\prime} G \otimes \mathscr{O}_{X}\right) \stackrel{\sim}{\sim} \mathscr{H}_{0} m_{\mathscr{D}_{X}}\left(\mathscr{M} \otimes G, \mathscr{O}_{X}\right)
$$

Assume moreover that $\operatorname{Supp}(\mathscr{M}) \cap \operatorname{Supp}(G)$ is compact. Applying Corollary 1.5 .2 (b), we get that the cohomology of the complex

$$
\operatorname{Sol}(\mathscr{M} \otimes G):=\operatorname{RHom}_{\mathscr{D}_{X}}\left(\mathscr{M} \otimes G, \mathscr{O}_{X}\right)
$$

is finite dimensional. Moreover

$$
\mathrm{R} \Gamma(X \times X ; \operatorname{TK}(\mathscr{M}, G)) \simeq \operatorname{Sol}(\mathscr{M} \otimes G) \otimes \operatorname{Sol}(\mathscr{M} \otimes G)^{*} .
$$

Applying Proposition 2.3.2, we get

$$
\begin{aligned}
\chi\left(\mathrm{R}_{\mathscr{H} o m_{\mathscr{D}_{X}}}\left(\mathscr{M} \otimes G, \mathscr{O}_{X}\right)\right) & =\left.\int_{X}\left(\mathrm{hh}_{\mathscr{E}}(\mathscr{M}) \star \mu \mathrm{eu}_{X}(G)\right)\right|_{X} \\
& =\int_{T^{*} X}\left(\operatorname{hh}_{\mathscr{E}}(\mathscr{M}) \cup \mu \mathrm{eu}_{X}(G)\right) .
\end{aligned}
$$


This formula has many applications, as far as one is able to calculate $\mu \mathrm{eu}_{X}(\mathscr{M})$.

Assume that $\mathscr{M}$ is endowed with a good filtration and $\operatorname{char}(\mathscr{M}) \subset \Lambda$. Set

$$
\begin{aligned}
& \widetilde{\operatorname{gr} \mathscr{M}}:=\mathscr{O}_{T^{*} X} \otimes_{\pi^{-1} \operatorname{gr} \mathscr{D} X} \pi^{-1} \operatorname{gr} \mathscr{M} \\
& \sigma_{\Lambda}(\mathscr{M})=\operatorname{Ch}_{\Lambda}(\widetilde{\mathrm{gr}} \mathscr{M}) \in \bigoplus_{j} H_{\Lambda}^{2 j}\left(T^{*} X ; \mathbb{C}_{T^{*} X}\right) \\
& \mu \operatorname{Ch}_{\Lambda}(\mathscr{M})=\sigma_{\Lambda}(\mathscr{M}) \cup \pi^{*} \operatorname{Td}_{X}\left(T^{*} X\right) \text { for a left } \mathscr{D} \text {-module, } \\
& \mu \operatorname{Ch}_{\Lambda}(\mathscr{M})=\sigma_{\Lambda}(\mathscr{M}) \cup \pi^{*} \operatorname{Td}_{X}(T X) \text { for a right } \mathscr{D} \text {-module, }
\end{aligned}
$$

where $\mathrm{Ch}$ is the Chern character and $\mathrm{Td}$ is the Todd class. Note that $\mu \mathrm{Ch}$ commutes with proper direct images (Laumon's version of the RR theorem for $\mathscr{D}$-modules) and non characteristic inverse images. In [ScSn94] we made the conjecture that

$$
\mu \mathrm{eu}_{\Lambda}(\mathscr{M})=\left[\mu \mathrm{Ch}_{\Lambda}(\mathscr{M})\right]^{2 d_{X}}
$$

This conjecture has been proved in BNT02 by Bressler-Nest-Tsygan and generalized in [BGNT07].

Example 2.6.1. (i) If $X$ is a complex compact manifold, one recovers the Riemann-Roch theorem: one takes $G=\mathbb{C}_{X}$ and if $\mathscr{F}$ is a coherent $\mathscr{O}_{X^{-}}$ module, one sets $\mathscr{M}=\mathscr{D}_{X} \otimes_{\mathscr{O}_{X}} \mathscr{F}$.

(ii) If $M$ is a compact real analytic manifold and $X$ is a complexification of $M$, one recovers the Atiyah-Singer theorem by choosing $G=\mathrm{D}_{X}^{\prime} \mathbb{C}_{M}$. 


\title{
Lecture 3
}

\section{Ind-sheaves and applications to $\mathscr{D}$-modules}

\begin{abstract}
I will first recall the constructions of [KS96, KS01] of the sheaves of temperate or Whitney holomorphic functions. These are not sheaves on the usual topology, but sheaves on the subanalytic site or better, ind-sheaves. Then I will explain how these objects appear naturally in the study of irregular holonomic $\mathscr{D}$-modules.
\end{abstract}

\subsection{Ind-sheaves}

\section{Ind-objects}

References are made to [SGA4] or to [KS06] for an exposition. We keep the notations of the preceding lectures.

Let $\mathscr{C}$ be an abelian category (in a given universe $\mathscr{U}$ ). One denotes by $\mathscr{C}^{\wedge, \text { add }}$ the big category of additive functors from $\mathscr{C}^{\text {op }}$ to $\operatorname{Mod}(\mathbb{Z})$. This big category is abelian and the functor $h^{\wedge}: \mathscr{C} \rightarrow \mathscr{C}^{\wedge}$ makes $\mathscr{C}$ a full abelian subcategory of $\mathscr{C}^{\wedge, a d d}$. This functor is left exact, but not exact in general.

An ind-object in $\mathscr{C}$ is an object $A \in \mathscr{C}^{\wedge}$ which is isomorphic to "lim" $\alpha$ for some functor $\alpha: I \rightarrow \mathscr{C}$ with $I$ filtrant and small. One denotes by $\operatorname{Ind}(\mathscr{C})$ the full additive subcategory of $\mathscr{C}^{\wedge, \text { add }}$ consisting of ind-objects.

Theorem 3.1.1. (i) The category $\operatorname{Ind}(\mathscr{C})$ is abelian.

(ii) The natural functor $\mathscr{C} \rightarrow \operatorname{Ind}(\mathscr{C})$ is fully faithful and exact and the natural functor $\operatorname{Ind}(\mathscr{C}) \rightarrow \mathscr{C}^{\wedge, \text { add }}$ is fully faithful and left exact.

(iii) The category $\operatorname{Ind}(\mathscr{C})$ admits exact small filtrant inductive limits and the functor $\operatorname{Ind}(\mathscr{C}) \rightarrow \mathscr{C}^{\wedge, \text { add }}$ commutes with such limits. 
(iv) Assume that $\mathscr{C}$ admits small projective limits. Then the category $\operatorname{Ind}(\mathscr{C})$ admits small projective limits, and the functor $\mathscr{C} \rightarrow \operatorname{Ind}(\mathscr{C})$ commutes with such limits.

Example 3.1.2. Assume that $\mathbf{k}$ is a field and denote by $\operatorname{Mod}^{\mathrm{f}}(\mathbf{k})$ the category of finite dimensional $\mathbf{k}$-vector spaces. Let $\mathrm{I}(\mathbf{k})$ denote the category of ind-objects of $\operatorname{Mod}(\mathbf{k})$. Define $\beta: \operatorname{Mod}(\mathbf{k}) \rightarrow \mathrm{I}(\mathbf{k})$ by setting $\beta(V)=$ "lim" $W$, where $W$ ranges over the family of finite-dimensional vector subspaces of $V$. In other words, $\beta(V)$ is the functor from $\operatorname{Mod}(\mathbf{k})^{\text {op }}$ to $\operatorname{Mod}(\mathbb{Z})$ given by $M \mapsto \underset{W}{\lim _{W}} \operatorname{Hom}_{k}(M, W)$. Therefore,

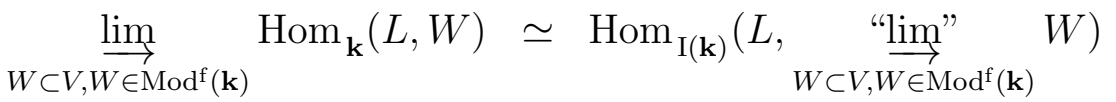

$$
\begin{aligned}
& =\operatorname{Hom}_{\mathrm{I}(\mathbf{k})}(L, \beta(V)) \text {. }
\end{aligned}
$$

If $V$ is infinite-dimensional, $\beta(V)$ is not representable in $\operatorname{Mod}(\mathbf{k})$. Moreover, $\operatorname{Hom}_{\mathrm{I}(\mathbf{k})}(\mathbf{k}, V / \beta(V)) \simeq 0$.

It is proved in $\mathrm{KS} 06$ that the category $\operatorname{Ind}(\mathscr{C})$ for $\mathscr{C}=\operatorname{Mod}(\mathbf{k})$ does not have enough injectives.

Definition 3.1.3. An object $A \in \operatorname{Ind}(\mathscr{C})$ is quasi-injective if the functor $\operatorname{Hom}_{\operatorname{Ind}(\mathscr{C})}(\bullet, A)$ is exact on the category $\mathscr{C}$.

It is proved in loc. cit. that if $\mathscr{C}$ has enough injectives, then $\operatorname{Ind}(\mathscr{C})$ has enough quasi-injectives.

\section{Ind-sheaves}

References are made to KS01.

Let $X$ be a locally compact space countable at infinity. Recall that $\operatorname{Mod}\left(\mathbf{k}_{X}\right)$ denotes the abelian category of sheaves of $\mathbf{k}$-modules on $X$. We denote by $\operatorname{Mod}^{\mathrm{c}}\left(\mathbf{k}_{X}\right)$ the full subcategory consisting of sheaves with compact support. We set for short:

$$
\mathrm{I}\left(\mathbf{k}_{X}\right):=\operatorname{Ind}\left(\operatorname{Mod}^{\mathrm{c}}\left(\mathbf{k}_{X}\right)\right)
$$

and call an object of this category an indsheaf on $X$.

Theorem 3.1.4. The prestack $U \mapsto \mathrm{I}\left(\mathbf{k}_{U}\right), U$ open in $X$ is a stack.

The following example explains why we have considered sheaves with compact supports. 
Example 3.1.5. Let $X=\mathbb{R}$, let $F=\mathbf{k}_{X}, G_{n}=\mathbf{k}_{[n,+\infty}, G=\underset{n}{\stackrel{\lim }{\longrightarrow}} G_{n}$. Then $\left.G\right|_{U}=0$ in $\operatorname{Ind}\left(\operatorname{Mod}\left(\mathbf{k}_{U}\right)\right)$ for any relatively compact open subset $U$ of $X$. On the other hand, $\operatorname{Hom}_{\operatorname{Ind}\left(\operatorname{Mod}\left(\mathbf{k}_{X}\right)\right)}\left(\mathbf{k}_{X}, G\right) \simeq \underset{n}{\lim } \operatorname{Hom}_{\mathbf{k}_{X}}\left(\mathbf{k}_{X}, G_{n}\right) \simeq \mathbf{k}$.

We have two pairs $\left(\alpha_{X}, \iota_{X}\right)$ and $\left(\beta_{X}, \alpha_{X}\right)$ of adjoint functors

$$
\operatorname{Mod}\left(\mathbf{k}_{X}\right) \underset{\beta_{X}}{\stackrel{\iota_{X}}{\rightleftarrows}} \mathrm{I}\left(\mathbf{k}_{X}\right) .
$$

The functor $\iota_{X}$ is the natural one. If $F$ has compact support, $\iota_{X}(F)=F$ after identifying a category $\mathscr{C}$ to a full subcategory of $\operatorname{Ind}(\mathscr{C})$. The functor $\alpha_{X}$ associates $\underset{i}{\lim } F_{i}\left(F_{i} \in \operatorname{Mod}^{\mathrm{c}}\left(\mathbf{k}_{X}\right), i \in I, I\right.$ small and filtrant) to the object $\underset{i}{\stackrel{\lim }{\longrightarrow}} F_{i}$. If $\mathbf{k}$ is a field, $\beta_{X}(F)$ is the functor $G \mapsto \Gamma\left(X ; H^{0}\left(\mathrm{D}_{X}^{\prime} G\right) \otimes F\right)$.

- $\iota_{X}$ is exact, fully faithful, and commutes with $\lim _{\longleftarrow}$,

- $\alpha_{X}$ is exact and commutes with $\underset{\leftarrow}{\longleftarrow}$ and $\underline{\lim }$,

- $\beta_{X}$ is right exact, fully faithful and commutes with $\stackrel{\lim }{\longrightarrow}$,

- $\alpha_{X}$ is left adjoint to $\iota_{X}$,

- $\alpha_{X}$ is right adjoint to $\beta_{X}$,

- $\alpha_{X} \circ \iota_{X} \simeq \operatorname{id}_{\operatorname{Mod}\left(\mathbf{k}_{X}\right)}$ and $\alpha_{X} \circ \beta_{X} \simeq \operatorname{id}_{\operatorname{Mod}\left(\mathbf{k}_{X}\right)}$.

Example 3.1.6. Let $U \subset X$ be an open subset, $S \subset X$ a closed subset. Then

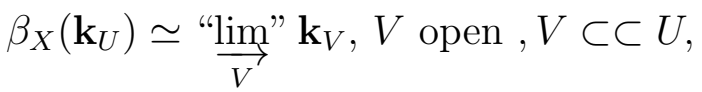

$$
\begin{aligned}
& \beta_{X}\left(\mathbf{k}_{S}\right) \simeq \underset{V}{\underset{V}{\longrightarrow}} \mathbf{k}_{\bar{V}}, V \text { open }, S \subset V \text {. }
\end{aligned}
$$

Let $a \in X$ and consider the skyscraper sheaf $\mathbf{k}_{\{a\}}$. Then $\beta_{X}\left(\mathbf{k}_{\{a\}}\right) \rightarrow \mathbf{k}_{\{a\}}$ is an epimorphism in $\mathrm{I}\left(\mathbf{k}_{X}\right)$ and defining $N_{a}$ by the exact sequence:

$$
0 \rightarrow N_{a} \rightarrow \beta_{X}\left(\mathbf{k}_{\{a\}}\right) \rightarrow \mathbf{k}_{\{a\}} \rightarrow 0
$$

we get that $\operatorname{Hom}_{\mathrm{I}\left(\mathbf{k}_{X}\right)}\left(\mathbf{k}_{U}, N_{a}\right) \simeq 0$ for all open neighborhood $U$ of $a$.

We shall not recall here the construction of the derived category of indsheaves, nor the six operations on such "sheaves". 


\subsection{Sheaves on the subanalytic site}

The subanalytic site was introduced in [KS01, Chapt 7] and the results on sheaves on this site were obtained as particular cases of more general results on indsheaves, which makes the reading not so easy. A direct and more elementary study of sheaves on the subanalytic site is performed in $\operatorname{Pr} 08$, Pr12.

Let $M$ be a real analytic manifold. One denotes by $\mathbb{R}-\mathrm{C}\left(\mathbf{k}_{M}\right)$ the abelian category of $\mathbb{R}$-constructible sheaves on $M$ and by $\mathbb{R}-C^{c}\left(\mathbf{k}_{M}\right)$ the full subcategory consisting of sheaves with compact support. There is an equivalence $\mathrm{D}^{\mathrm{b}}\left(\mathbb{R}-\mathrm{C}\left(\mathbf{k}_{M}\right)\right) \simeq \mathrm{D}_{\mathbb{R}_{-} \mathrm{c}}^{\mathrm{b}}\left(\mathbf{k}_{M}\right)$ where this last category is the full triangulated subcategory of $\mathrm{D}^{\mathrm{b}}\left(\mathbf{k}_{M}\right)$ consisting of $\mathbb{R}$-constructible sheaves. (This classical result has first been proved by Kashiwara [Ka84].)

We denote by $\mathrm{Op}_{M}$ the category whose objects are the open subsets of $M$ and the morphisms are the inclusions of open subsets. One defines a Grothendieck topology on $\mathrm{Op}_{M}$ by deciding that a family $\left\{U_{i}\right\}_{i \in I}$ of subobjects of $U \in \mathrm{Op}_{M}$ is a covering of $U$ if it is a covering in the usual sense.

Definition 3.2.1. Denote by $\mathrm{Op}_{M_{\mathrm{sa}}}$ the full subcategory of $\mathrm{Op}_{M}$ consisting of subanalytic and relatively compact open subsets. The site $M_{\mathrm{sa}}$ is obtained by deciding that a family $\left\{U_{i}\right\}_{i \in I}$ of subobjects of $U \in \mathrm{Op}_{M_{\mathrm{sa}}}$ is a covering of $U$ if there exists a finite subset $J \subset I$ such that $\bigcup_{j \in J} U_{j}=U$.

Let us denote by

$$
\rho_{\mathrm{sa}}: M \rightarrow M_{\mathrm{sa}}
$$

the natural morphism of sites. Here again, we have two pairs of adjoint functors $\left(\rho_{\mathrm{sa}}^{-1}, \rho_{\mathrm{sa} *}\right)$ and $\left(\rho_{\mathrm{sa} !}, \rho_{\mathrm{sa}}^{-1}\right)$ :

$$
\operatorname{Mod}\left(\mathbf{k}_{M}\right) \underset{\rho_{\mathrm{sa} *}}{\stackrel{\rho_{\mathrm{sa}}^{-1} \longrightarrow}{\longleftarrow}} \operatorname{\rho _{\mathrm {sa}!}} \operatorname{Mod}\left(\mathbf{k}_{M_{\mathrm{sa}}}\right) .
$$

For $F \in \operatorname{Mod}\left(\mathbf{k}_{M}\right), \rho_{\mathrm{sa}} F$ is the sheaf associated to the presheaf $U \mapsto F(\bar{U})$, $U \in \mathrm{Op}_{M_{\mathrm{sa}}}$.

Proposition 3.2.2. The restriction of the functor $\rho_{\mathrm{sa} *}$ to the category $\mathbb{R}-\mathrm{C}\left(\mathbf{k}_{M}\right)$ is exact and fully faithful.

By this result, we shall consider the category $\mathbb{R}-\mathrm{C}\left(\mathbf{k}_{M}\right)$ as a full subcate$\operatorname{gory}$ of $\operatorname{Mod}\left(\mathbf{k}_{M}\right)$ as well as a full subcategory of $\operatorname{Mod}\left(\mathbf{k}_{M_{\mathrm{sa}}}\right)$. Set

$$
\mathrm{I}_{\mathbb{R}-\mathrm{c}}\left(\mathbf{k}_{M}\right)=\operatorname{Ind}\left(\mathbb{R}-\mathrm{C}^{c}\left(\mathbf{k}_{M}\right)\right) .
$$


Theorem 3.2.3. The natural functor $\alpha_{M_{\mathrm{sa}}}: \mathrm{I}_{\mathbb{R}-\mathrm{c}}\left(\mathbf{k}_{M}\right) \rightarrow \operatorname{Mod}\left(\mathbf{k}_{M_{\mathrm{sa}}}\right)$ is an equivalence of categories.

In other words, ind- $\mathbb{R}$-constructible sheaves are "usual sheaves" on the subanalytic site. By this result, the embedding $\mathbb{R}-\mathrm{C}^{c}\left(\mathbf{k}_{M}\right) \hookrightarrow \operatorname{Mod}^{\mathrm{c}}\left(\mathbf{k}_{M}\right)$ gives a functor $I_{M}: \operatorname{Mod}\left(\mathbf{k}_{M_{\mathrm{sa}}}\right) \rightarrow \mathrm{I}\left(\mathbf{k}_{M}\right)$. Hence, we have a quasi-commutative diagram of categories

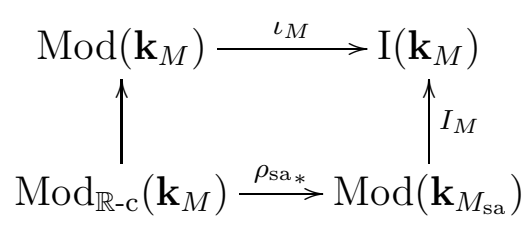

in which all arrows are exact and fully faithful. One shall be aware that the diagram:

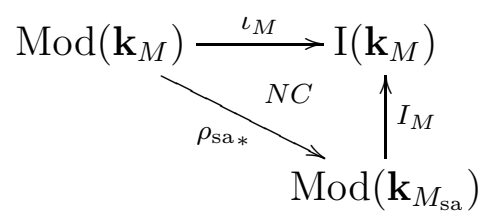

is not commutative. Moreover, $\iota_{M}$ is exact and $\rho_{\mathrm{sa} *}$ is not right exact in general.

One denotes by "lim" the inductive limit in the category $\operatorname{Mod}\left(\mathbf{k}_{M_{\mathrm{sa}}}\right)$. One shall be aware that the functor $I_{M}$ commutes with inductive limits but $\rho_{\mathrm{sa} *}$ does not.

\subsection{Moderate and formal cohomology}

From now on, $\mathbf{k}=\mathbb{C}$. As usual, we denote by $\mathscr{C}_{M}^{\infty}$ (resp. $\mathscr{C}_{M}^{\omega}$ ) the sheaf of complex functions of class $C^{\infty}$ (resp. real analytic), by $\mathscr{D} b_{M}$ (resp. $\mathscr{B}_{M}$ ) the sheaf of Schwartz's distributions (resp. Sato's hyperfunctions), and by $\mathscr{D}_{M}$ the sheaf of analytic finite-order differential operators. We also use the notation $\mathscr{A}_{M}=\mathscr{C}_{M}^{\omega}$.

Definition 3.3.1. Let $U \in \mathrm{Op}_{M_{\mathrm{sa}}}$ and let $f \in \mathscr{C}_{M}^{\infty}(U)$. One says that $f$ has polynomial growth at $p \in M$ if it satisfies the following condition. For a local coordinate system $\left(x_{1}, \ldots, x_{n}\right)$ around $p$, there exist a sufficiently small compact neighborhood $K$ of $p$ and a positive integer $N$ such that

$$
\sup _{x \in K \cap U}(\operatorname{dist}(x, K \backslash U))^{N}|f(x)|<\infty .
$$


It is obvious that $f$ has polynomial growth at any point of $U$. We say that $f$ is temperate at $p$ if all its derivatives have polynomial growth at $p$. We say that $f$ is temperate if it is temperate at any point.

For $U \in \mathrm{Op}_{M_{\mathrm{sa}}}$, denote by $\mathcal{C}_{M}^{\infty, \mathrm{tp}}(U)$ the subspace of $\mathscr{C}_{M}^{\infty}(U)$ consisting of tempered functions.

Denote by $\mathcal{D} b_{M}^{\mathrm{tp}}(U)$ the space of tempered distributions on $U$, defined by the exact sequence

$$
0 \rightarrow \Gamma_{M \backslash U}\left(M ; \mathcal{D} b_{M}\right) \rightarrow \Gamma\left(M ; \mathscr{D} b_{M}\right) \rightarrow \mathcal{D} b_{M}^{\mathrm{tp}}(U) \rightarrow 0 .
$$

Using Lojasiewicz's inequalities [Lo61] (see also [Ma67]), one easily proves that

- the presheaf $U \mapsto \mathcal{C}_{M}^{\infty, t p}(U)$ is a sheaf on $M_{\mathrm{sa}}$,

- the presheaf $U \mapsto \mathcal{D} b_{M}^{\mathrm{tp}}(U)$ is a sheaf on $M_{\mathrm{sa}}$.

One denotes by $\mathcal{C}_{M_{\mathrm{sa}}}^{\infty, \mathrm{tp}}$ the first one and calls it the sheaf of temperate $C^{\infty}$ functions. One denotes by $\mathcal{D} b_{M_{\mathrm{sa}}}^{\mathrm{tp}}$ the second one and calls it the sheaf of temperate distributions. Let $F \in \mathrm{D}_{\mathbb{R}-\mathrm{c}}^{\mathrm{b}}\left(\mathbb{C}_{M}\right)$. One has the isomorphism

$$
\rho_{\mathrm{sa}}^{-1} \operatorname{R} \mathscr{H} o m\left(F, \mathcal{D} b_{M_{\mathrm{sa}}}^{\mathrm{tp}}\right) \simeq \operatorname{thom}\left(F, \mathcal{D} b_{M}\right)
$$

where the right-hand side was defined by Kashiwara as the main tool for his proof of the Riemann-Hilbert correspondence in [Ka80, Ka84].

For a closed subanalytic subset $S$ in $M$, denote by $\mathscr{I}_{M, S}^{\infty}$ the subsheaf of $\mathscr{C}_{M}^{\infty}$ consisting of functions which vanish up to infinite order on $S$. In [KS96], one introduces the sheaf:

$$
\mathbb{C}_{U} \stackrel{\mathrm{w}}{\otimes} \mathscr{C}_{M}^{\infty}:=V \mapsto \Gamma\left(V ; \mathscr{I}_{V, V \backslash U}^{\infty}\right)
$$

and shows how to extend this construction and define an exact functor $\bullet \stackrel{\mathrm{w}}{\otimes} \mathscr{C}_{M}^{\infty}$ on $\operatorname{Mod}_{\mathbb{R}-\mathrm{c}}\left(\mathbb{C}_{M}\right)$. One denotes by $\mathscr{C}_{M}^{\infty, \mathrm{w}}$ the sheaf on $M_{\mathrm{sa}}$ given by

$$
\mathscr{C}_{M}^{\infty, \mathrm{w}}(U)=\Gamma\left(M ; H^{0}\left(\mathrm{D}_{M}^{\prime} \mathbf{k}_{U}\right) \stackrel{\mathrm{w}}{\otimes} \mathscr{C}_{M}^{\infty}\right), U \in \mathrm{Op}_{M_{\mathrm{sa}}}
$$

If $\mathrm{D}_{M}^{\prime} \mathbb{C}_{U} \simeq \mathbb{C}_{\bar{U}}, \mathscr{C}_{M}^{\infty, \mathrm{w}}(U)$ is the space of Whitney functions on $U$, that is the quotient $\mathscr{C}^{\infty}(M) / \mathscr{I}_{M, M \backslash U}^{\infty}$. It is thus natural to call $\mathscr{C}_{M}^{\infty}$,w the sheaf of Whitney $C^{\infty}$-functions on $M_{\text {sa }}$.

Note that the sheaf $\rho_{\mathrm{sa*}} \mathscr{D}_{M}$ does not operate on the sheaves $\mathcal{C}_{M}^{\infty, \text { tp }}, \mathscr{D} b_{M}^{t}$, $\mathbb{C}_{M}^{\infty, \mathrm{w}}$ but $\rho_{\mathrm{sa} !} \mathscr{D}_{M}$ does. 
Now let $X$ be a complex manifold. We still denote by $X$ the real underlying manifold and we denote by $\bar{X}$ the complex manifold conjugate to $X$. One defines the sheaf of temperate holomorphic functions $\mathcal{O}_{X_{\mathrm{sa}}}^{\mathrm{t} p}$ as the Dolbeault complex with coefficients in $\mathcal{C}_{X_{\mathrm{sa}}}^{\infty, t p}$. More precisely

$$
\mathcal{O}_{X_{\mathrm{sa}}}^{\mathrm{t} p}=\mathrm{R} \mathscr{H} O m_{\rho_{\mathrm{sa} !} \mathscr{\mathscr { P } _ { X }}}\left(\rho_{\mathrm{sa} !} \mathscr{O}_{\bar{X}}, \mathcal{C}_{X_{\mathrm{sa}}}^{\infty, \mathrm{tp}}\right) .
$$

One proves the isomorphism

$$
\mathcal{O}_{X_{\mathrm{sa}}}^{\mathrm{t} p} \simeq \mathrm{R} \mathscr{H}_{O m_{\rho_{\mathrm{sa} !} \mathscr{Q}}}\left(\rho_{\mathrm{sa} !} \mathscr{O}_{\bar{X}}, \mathcal{D} b_{X_{\mathrm{sa}}}^{\mathrm{tp}}\right) .
$$

Similarly, one defines the sheaf

$$
\mathcal{O}_{X_{\mathrm{sa}}}^{\mathrm{w}}=\mathrm{R} \mathscr{H} O m_{\rho_{\mathrm{sa} !} \mathscr{O}_{\bar{X}}}\left(\rho_{\mathrm{sa} !} \mathscr{O}_{\bar{X}}, \mathscr{C}_{X_{\mathrm{sa}}}^{\infty, \mathrm{w}}\right)
$$

Note that the objects $\mathcal{O}_{X_{\mathrm{sa}}}^{\mathrm{t} p}$ and $\mathcal{O}_{X_{\mathrm{sa}}}^{\mathrm{w}}$ are not concentrated in degree zero in dimension $>1$. Indeed, with the subanalytic topology, only finite coverings are allowed. If one considers for example the open set $U \subset \mathbb{C}^{n}$, the difference of a open ball of radius $R>0$ and a closed ball of radius $r$ with $0<r<R$, then the Dolbeault complex will not be exact after any finite covering. For the same reason, the sheaf $\mathrm{R} \rho_{\mathrm{sa} *} \mathscr{O}_{X}$ is not concentrated in degree zero in dimension $>1$.

Therefore, we shall better consider indsheaves and we shall embed the category $\mathrm{D}^{\mathrm{b}}\left(\mathbb{C}_{X_{\mathrm{sa}}}\right)$ into the category $\mathrm{D}^{\mathrm{b}}\left(\mathrm{I}\left(\mathbb{C}_{X}\right)\right)$ by the exact functor $I_{X}$. Hence we consider subanalytic sheaves as indsheaves. In the category $\mathrm{D}^{\mathrm{b}}\left(\mathrm{I}\left(\mathbb{C}_{X}\right)\right)$ we have thus the morphisms of sheaves

$$
\mathcal{O}_{X}^{\omega} \rightarrow \mathcal{O}_{X}^{\mathrm{w}} \rightarrow \mathcal{O}_{X}^{\mathrm{t} p} \rightarrow \mathscr{O}_{X}
$$

Here $\mathcal{O}_{X}^{\mathrm{w}}$ and $\mathcal{O}_{X}^{\mathrm{t} p}$ are the images of $\mathcal{O}_{X_{\mathrm{sa}}}^{\mathrm{w}}$ and $\mathcal{O}_{X_{\mathrm{sa}}}^{\mathrm{t} p}$ by the functor $I_{X}$ (there are still not concentrated in degree 0$)$, we have kept the same notation for $\mathscr{O}_{X}$ and its image in $\operatorname{Mod}\left(\mathrm{I}\left(\mathbb{C}_{X}\right)\right)$ by the functor $\iota_{X}$, and we have set

$$
\mathcal{O}_{X}^{\omega}:=\beta_{X}\left(\mathscr{O}_{X}\right)
$$

We call $\mathcal{O}_{X}^{\mathrm{w}}$ and $\mathcal{O}_{X}^{\mathrm{t} p}$ the sheaves of temperate and Whitney holomorphic functions, respectively.

Example 3.3.2. Let $Z$ be a closed complex analytic subset of the complex manifold $X$. We have the isomorphisms

$$
\begin{aligned}
& \left.\alpha_{X} \mathrm{R} \mathscr{H}_{\mathrm{I}\left(\mathbb{C}_{X}\right)}\left(\mathrm{D}^{\prime} \mathbb{C}_{Z}, \mathcal{O}_{X}^{\omega}\right) \simeq \mathscr{O}_{X}\right|_{Z}, \\
& \alpha_{X} \mathrm{R} \mathscr{H} \operatorname{om}_{\mathrm{I}\left(\mathbb{C}_{X}\right)}\left(\mathrm{D}^{\prime} \mathbb{C}_{Z}, \mathcal{O}_{X}^{\mathrm{w}}\right) \simeq \mathscr{O}_{X} \widehat{\mid}_{Z} \text { (formal completion along } Z \text { ), } \\
& \alpha_{X} \mathrm{R} \mathscr{H}_{\text {om }}{\operatorname{I(\mathbb {C}_{X})}}\left(\mathbb{C}_{Z}, \mathcal{O}_{X}^{\mathrm{t} p}\right) \simeq \mathrm{R} \Gamma_{[Z]}\left(\mathscr{O}_{X}\right) \text { (algebraic cohomology), } \\
& \alpha_{X} \mathrm{R} \mathscr{H} o m_{\mathrm{I}\left(\mathbb{C}_{X}\right)}\left(\mathbb{C}_{Z}, \mathscr{O}_{X}\right) \simeq \mathrm{R} \Gamma_{Z}\left(\mathscr{O}_{X}\right) \text {. }
\end{aligned}
$$


Example 3.3.3. let $M$ be a real analytic manifold and $X$ a complexification of $M$. We have the isomorphisms

$$
\begin{aligned}
& \alpha_{X} \operatorname{RH} \mathscr{H o m}_{\mathrm{I}\left(\mathbb{C}_{X}\right)}\left(\mathrm{D}^{\prime} \mathbb{C}_{M}, \mathcal{O}_{X}^{\omega}\right) \simeq \mathscr{A}_{M}, \\
& \left.\alpha_{X} \mathrm{R} \mathscr{H}_{\text {om }} \operatorname{IC}_{X}\right)\left(\mathrm{D}^{\prime} \mathbb{C}_{M}, \mathcal{O}_{X}^{\mathrm{w}}\right) \simeq \mathscr{C}_{M}^{\infty} \text {, } \\
& \alpha_{X} \mathrm{R} \mathscr{H o m}_{\mathrm{I}\left(\mathbb{C}_{X}\right)}\left(\mathrm{D}^{\prime} \mathbb{C}_{M}, \mathcal{O}_{X}^{\mathrm{t} p}\right) \simeq \mathcal{D} b_{M}, \\
& \alpha_{X} \mathrm{R} \mathscr{H}_{\text {om }}\left(\mathbb{C}_{X}\right)\left(\mathrm{D}^{\prime} \mathbb{C}_{M}, \mathscr{O}_{X}\right) \simeq \mathscr{B}_{M} \text {. }
\end{aligned}
$$

Notice that with this approach, the sheaf $\mathcal{D} b_{M}$ of Schwartz's distributions is constructed similarly as the sheaf of Sato's hyperfunctions. In particular, functional analysis is not used in the construction.

Remark 3.3.4. The subanalytic topology allows us to define functions whose growth at the boundary is bounded by some power of the inverse of the distance to the boundary, but not to make precise this power. In order to define such sheaves, we have recently defined with S. Guillermou in [GS13] the linear subanalytic topology $M_{\text {sal }}$ on a real analytic manifold $M$. The open sets of this topology are those of $M_{\mathrm{sa}}$, namely $\mathrm{Op}_{M_{\mathrm{sa}}}$, but there are less coverings. Roughly speaking, a finite covering $\left\{U_{i}\right\}_{i \in I}$ is a linear covering of $U=\bigcup_{i} U_{i}$ if there is a constant $C$ such that for any $x \in M$

$$
d\left(x, M \backslash \bigcup_{i \in I} U_{i}\right) \leq C \cdot \max _{i \in I} d\left(x, M \backslash U_{i}\right) .
$$

Here $d$ is a distance on $M$ which is locally equivalent to the Euclidian distance on $\mathbb{R}^{n}$. One proves that the family of linear coverings satisfies the axioms of Grothendieck topologies. One denotes by $M_{\text {sal }}$ the site so defined and by $\rho_{\mathrm{sal}}: M_{\mathrm{sa}} \rightarrow M_{\mathrm{sal}}$ the natural morphism of sites. One of the main results of the theory is that functor $\mathrm{R} \rho_{\text {sal } *}: \mathrm{D}^{+}\left(\mathbf{k}_{M_{\mathrm{sa}}}\right) \rightarrow \mathrm{D}^{+}\left(\mathbf{k}_{M_{\mathrm{sal}}}\right)$ admits a right adjoint $\rho_{\text {sal }}^{!}: \mathrm{D}^{+}\left(\mathbf{k}_{M_{\mathrm{sal}}}\right) \rightarrow \mathrm{D}^{+}\left(\mathbf{k}_{M_{\mathrm{sa}}}\right)$. Moreover, if $U \in \mathrm{Op}_{M_{\mathrm{sa}}}$ has Lipschitz boundary, then $\mathrm{R} \rho_{\text {sal }} \mathbb{C}_{U}$ is concentrated in degree 0 . It follows that if $F$ is a presheaf on $M_{\text {sa }}$ such that the sequence $0 \rightarrow F\left(U_{1} \cup U_{2}\right) \rightarrow F\left(U_{1}\right) \oplus F\left(U_{2}\right) \rightarrow$ $F\left(U_{1} \cap U_{2}\right) \rightarrow 0$ is exact for any linear covering $\left(U_{1}, U_{2}\right)$ of $U_{1} \cup U_{2}$, then there exists $F \in \mathrm{D}^{+}\left(\mathbf{k}_{M_{\mathrm{sa}}}\right)$ such that $\mathrm{R} \Gamma(U ; F) \simeq F(U)$ for all $U \in \mathrm{Op}_{M_{\mathrm{sa}}}$ with Lipchitz boundaries.

This topology allows us to define the subsheaf $\mathcal{C}_{M_{\text {sal }}}^{\infty, s}$ of $\mathcal{C}_{M_{\text {sal }}}^{\infty}$ consisting of functions tempered of order $s$. On a complex manifold $X$ we may thus endow the sheaf $\mathcal{O}_{X_{\mathrm{sa}}}^{\mathrm{p}}$ with a natural filtration (in the derived sense). We refer to loc. cit. more more details. 


\subsection{Applications to $\mathscr{D}$-modules I}

Let us show on an example extracted of [KS03] the possible role of the sheaf $\mathscr{O}_{X}^{t}$ in the study of irregular holonomic $\mathscr{D}$-modules.

Let $X$ be a complex manifold and let $\mathscr{M}$ be a holonomic $\mathscr{D}$-module. We set for short

$$
\begin{aligned}
\operatorname{Sol}^{0}(\mathscr{M}) & =\mathscr{H} m_{\mathscr{D}_{X}}\left(\mathscr{M}, \mathscr{O}_{X}\right), \\
\mathcal{S}_{o l} l^{0, t}(\mathscr{M}) & =\mathscr{H} m_{\beta_{X} \mathscr{D}_{X}}\left(\beta_{X} \mathscr{M}, \mathscr{O}_{X}^{t}\right)
\end{aligned}
$$

We shall compare these two objects in a simple example in which $\mathscr{M}$ is not regular. Let $X=\mathbb{C}$ endowed with the holomorphic coordinate $z$ and let $P=z^{2} \partial_{z}+1$. We consider the $\mathscr{D}_{X}$-module $\mathscr{M}:=\mathscr{D}_{X} \exp (1 / z) \simeq \mathscr{D}_{X} / \mathscr{D}_{X} \cdot P$.

Notice first that $\mathscr{O}_{X}^{t}$ is concentrated in degree 0 (since $\operatorname{dim} X=1$ ) and it is a sub-indsheaf of $\mathscr{O}_{X}$. It follows that the morphism $\mathcal{S}_{0} l^{0, t}(\mathscr{M}) \rightarrow \mathcal{S}_{0} l^{0}(\mathscr{M})$ is a monomorphism. Moreover,

$$
\operatorname{Sol}^{0}(\mathscr{M}) \simeq \mathbb{C}_{X, X \backslash\{0\}} \cdot \exp (1 / z) .
$$

It follows that for $V \subset X$ a connected open subset, we have $\Gamma\left(V ; \mathcal{S}_{0} l^{0, t}(\mathscr{M})\right) \neq$ 0 if and only if $V \subset X \backslash\{0\}$ and $\left.\exp (1 / z)\right|_{V}$ is tempered.

Let $\bar{B}_{\varepsilon}$ denote the closed ball with center $(\varepsilon, 0)$ and radius $\varepsilon$ and set $U_{\varepsilon}=X \backslash \bar{B}_{\varepsilon}$.

Then one proves that $\exp (1 / z)$ is temperate (in a neighborhood of 0 ) on an open subanalytic subset $V \subset X \backslash\{0\}$ if and only if $\operatorname{Re}(1 / z)$ is bounded on $V$, that is, if and only if $V \subset U_{\varepsilon}$ for some $\varepsilon>0$. We get

Proposition 3.4.1. One has the isomorphism

$$
\underset{\varepsilon>0}{\stackrel{\lim }{\longrightarrow}} \mathbb{C}_{X U_{\varepsilon}} \stackrel{\sim}{\longrightarrow} \mathcal{S}_{o l} l^{0, t}(\mathscr{M}) .
$$

Unfortunately, the functor $\mathcal{S}^{t}{ }^{t}$ (as well as its derived functor) is not fully faithful since the $\mathscr{D}$-modules $\mathscr{M}:=\mathscr{D}_{X} \exp (1 / z)$ and $\mathscr{N}:=\mathscr{D}_{X} \exp (2 / z)$ have the same indsheaves of temperate holomorphic solutions although they are not isomorphic.

Proposition 3.4.1 has been generalized to the study of holonomic modules in dimension one in Mr09.

\subsection{Applications to $\mathscr{D}$-modules II}

For $F \in \mathrm{D}_{\mathbb{R}-\mathrm{c}}^{\mathrm{b}}\left(\mathbb{C}_{X}\right)$, set $($ see $(\underline{3.3 .2}))$ :

$$
\begin{aligned}
F \stackrel{\mathrm{w}}{\otimes} \mathscr{O}_{X} & :=\operatorname{R}_{\mathscr{H} o m_{\mathscr{D}_{\bar{X}}}}\left(\mathscr{O}_{\bar{X}}, F \stackrel{\mathrm{w}}{\otimes} \mathscr{C}_{X}^{\infty}\right), \\
\operatorname{thom}\left(F, \mathscr{O}_{X}\right) & :=\operatorname{R}_{\mathscr{H} o m_{\mathscr{D}_{\bar{X}}}}\left(\mathscr{O}_{\bar{X}}, \operatorname{thom}\left(F, \mathcal{D} b_{X}\right)\right) .
\end{aligned}
$$


Let $F \in \mathrm{D}_{\mathbb{R - c}}^{\mathrm{b}}\left(\mathbb{C}_{X}\right)$ and $\mathscr{M} \in \mathrm{D}_{\text {coh }}^{\mathrm{b}}(\mathscr{D})$. Recall that we have set $\omega_{X}^{\text {hol }}:=$ $\Omega_{X}\left[d_{X}\right]$. Set for short

$$
\begin{aligned}
& W(\mathscr{M}, F):=\operatorname{R}_{\mathscr{H} o m_{\mathscr{D}}}\left(\mathscr{M}, F \stackrel{\mathrm{w}}{\otimes} \mathscr{O}_{X}\right), \\
& T(F, \mathscr{M}):=\operatorname{thom}\left(F, \omega_{X}^{\mathrm{hol}}\right) \stackrel{\mathrm{L}}{\otimes}_{\mathscr{D}} \mathscr{M} .
\end{aligned}
$$

There is a natural morphism

$$
W(\mathscr{M}, F) \otimes T(F, \mathscr{M}) \rightarrow \omega_{X}^{\text {hol }},
$$

functorial in $F$ and $\mathscr{M}$. For $G \in \mathrm{D}_{\mathbb{R}-\mathrm{c}}^{\mathrm{b}}\left(\mathbb{C}_{X}\right)$ one gets a pairing

$$
\begin{aligned}
\operatorname{RHom}(G, W(\mathscr{M}, F)) & \otimes \mathrm{R} \Gamma_{c}(X ; G \otimes T(F, \mathscr{M})) \\
& \rightarrow \mathrm{R} \Gamma_{c}(X ; W(\mathscr{M}, F) \otimes T(F, \mathscr{M})) \\
& \rightarrow \mathrm{R} \Gamma_{c}\left(X ; \omega_{X}^{\text {hol }}\right) \rightarrow \mathbb{C} .
\end{aligned}
$$

Denote by $\mathrm{D}^{\mathrm{b}}(F N)$ the derived category of the quasi-abelian category of Fréchet nuclear $\mathbb{C}$-vector spaces and define similarly the category $\mathrm{D}^{\mathrm{b}}(D F N)$, where now DFN stands for "dual of Fréchet nuclear".

Theorem 3.5.1. ([KS96, Theorem 6.1]) Let $F, G \in \mathrm{D}_{\mathbb{R}-c}^{\mathrm{b}}\left(\mathbb{C}_{X}\right)$ and $\mathscr{M} \in$ $\mathrm{D}_{\mathrm{coh}}^{\mathrm{b}}(\mathscr{D})$. Then the two complexes

$$
\operatorname{RHom}(G, W(\mathscr{M}, F)) \in \mathrm{D}^{\mathrm{b}}(F N) \text { and } \mathrm{R}_{c}(X ; G \otimes T(F, \mathscr{M})) \in \mathrm{D}^{\mathrm{b}}(D F N)
$$

are dual to each other through (3.5.2), functorially in $F, G$ and $\mathscr{M}$.

Now we assume that $\mathscr{M} \in \mathrm{D}_{\text {hol }}^{\mathrm{b}}\left(\mathscr{D}_{X}\right)$ and we consider the following assertions.

(a) $W(\mathscr{M}, F)=\mathrm{R} \mathscr{H} o m_{\mathscr{D}}\left(\mathscr{M}, F \stackrel{\mathrm{w}}{\otimes} \mathscr{O}_{X}\right)$ is $\mathbb{R}$-constructible,

(b) $T(F, \mathscr{M})=\operatorname{thom}\left(F, \omega_{X}^{\text {hol }}\right) \stackrel{\mathrm{L}}{\otimes_{\mathscr{D}}} \mathscr{M}$ is $\mathbb{R}$-constructible,

(c) the two complexes in (a) and (b) are dual to each other in the category $\mathrm{D}_{\mathbb{R}-\mathrm{c}}^{\mathrm{b}}\left(\mathbb{C}_{X}\right)$, that is, $W(\mathscr{M}, F) \simeq \mathrm{D}_{X} T(F, \mathscr{M})$.

It was conjectured in [KS03] that (b) is always satisfied. Based on the work of Mochizuki [Mo09] (see also [Ke10, Ke11, Sb12]), partial results in this direction have been obtained in Mr13.

On the other hand, one deduces easily from Theorem 3.5.1 that (a) and (b) are equivalent and imply (c). Finally, it follows immediately from [Ka78, Ka84 that (b), hence (a) and (c), are true when $F \in \mathrm{D}_{\mathbb{C}-\mathrm{c}}^{\mathrm{b}}\left(\mathbb{C}_{X}\right)$. 
Corollary 3.5.2. Assume that $F \in \mathrm{D}_{\mathbb{C}-c}^{\mathrm{b}}\left(\mathbb{C}_{X}\right)$ and $X$ is compact. Then the complexes $\mathrm{R} \Gamma(X ; W(\mathscr{M}, F))$ and $\mathrm{R} \Gamma(X ; T(F, \mathscr{M}))$ have finite-dimensional cohomology and (3.5.2) induces a perfect pairing for all $i \in \mathbb{Z}$

$$
H^{-i} \mathrm{R} \Gamma(X ; W(\mathscr{M}, F)) \otimes H^{i} \mathrm{R} \Gamma(X ; T(F, \mathscr{M})) \rightarrow \mathbb{C},
$$

functorial in $F$ and $\mathscr{M}$.

In BE04, S. Bloch and H. Esnault prove directly a similar result on an algebraic curve $X$ when assuming that $\mathscr{M}$ is a meromorphic connection with poles on a divisor $D$. They interpret the duality pairing by considering sections of the type $\gamma \otimes \varepsilon$, where $\gamma$ is a cycle with boundary on $D$ and $\varepsilon$ is a horizontal section of the connection on $\gamma$ with exponential decay on $D$. Their work has been extended to higher dimension by M. Hien [Hi09].

It would be interesting to make a link with these results and Corollary 3.5.2. 


\section{Bibliography}

[BE04] S. Bloch and H. Esnault, Homology for irregular connections, J. Théor. Nombres Bordeaux 16 p. 357-371 (2004).

[BGNT07] P. Bressler, A. Gorokhovsky, R. Nest and B. Tsygan, Deformation quantization of gerbes, Adv. Math. 214 p. 230-266 (2007).

[BNT02] P. Bressler, R. Nest and B. Tsygan, Riemann-Roch theorems via deformation quantization. I, II, Adv. Math. 167 p. 1-25, 26-73 (2002).

[BI73] J. Bros and D. Iagolnitzer, Causality and local analyticity: mathematical study, Annales Inst. Fourier 18 p. 147-184 (1973).

[BG87] J-L. Brylinski and E. Getzler, The homology of algebras of pseudodifferential symbols and the noncommutative residue, $K$-Theory 1 p. 385403 (1987).

[Ca05] A. Caldararu, The Mukai pairing II: the Hochschild-KostantRosenberg isomorphism, Adv. Math. 194 p. 34-66 (2005).

[Ca07] A. Caldararu and S. Willerton, The Mukai pairing I: a categorical approach, New York J. Math. 16 (2010).

arXiv:0707.2052

[FLTZ10] B. Fang, M. Liu, D. Treumann and E. Zaslow, The CoherentConstructible Correspondence and Fourier-Mukai Transforms, Acta Math. Sin. (Engl. Ser.) 27 no.2 (2011) 275-308.

arXiv: 1009.3506

[Ga81] O. Gabber, The integrability of the characteristic variety, Amer. Journ. Math. 103 (1981) 445-468.

[Gr09] J. Grivaux, On a conjecture of Kashiwara relating Chern and Euler classes of $\mathscr{O}$-modules, Journal of Diff. Geometry, (2012). arXiv:0910.5384 
[GS13] S. Guillermou and P. Schapira, Construction of sheaves on the subanalytic site, In preparation (2013). math.arXiv:1212.4326

[Hi09] M. Hien, Periods for flat algebraic connections, Invent. Math. 178 p. 1-22. (2009).

[Ho83] L. Hörmander, The analysis of linear partial differential operators, Grundlehren der Math. Wiss. 256 Springer-Verlag (1983).

[Hu06] D. Huybrechts, Fourier-Mukai transforms in algebraic geometry, Oxford Mathematical Monographs, Oxford (2006).

[Ka78] M. Kashiwara, On the holonomic systems of linear differential equations, II, Invent. Math. 49 p. 121-135, (1978).

[Ka80] _ Faisceaux constructibles et systèmes holonômes d'équations aux dérivées partielles linéaires à points singuliers réguliers, Séminaire Goulaouic-Schwartz, 1979-1980 (French), Exp. No. 19 École Polytech., Palaiseau, (1980).

[Ka84] _ The Riemann-Hilbert problem for holonomic systems, Publ. Res. Inst. Math. Sci. 20 p. 319-365, (1984).

[Ka85] _ Index theorem for constructible sheaves, Differential systems and singularities, Astrisque 130 Soc. Math. France p. 193209, (1985).

[Ka91] _ Letter to P. Schapira, unpublished, 18/11/1991.

[Ka03] _ D-modules and Microlocal Calculus, Translations of Mathematical Monographs, 217 American Math. Soc. (2003).

[KS82] M. Kashiwara and P. Schapira, Micro-support des faisceaux: applications aux modules différentiels, C. R. Acad. Sci. Paris série I Math 295 p. $487-490$ (1982).

[KS85] — Microlocal study of sheaves, Astérisque 128 Soc. Math. France (1985).

[KS90] _ Sheaves on Manifolds,

Grundlehren der Math. Wiss. 292 Springer-Verlag (1990).

[KS96] _ Moderate and formal cohomology associated with constructible sheaves, Mém. Soc. Math. France 64 (1996), iv+76 pp.

[KS97] _ Integral transforms with exponential kernels and Laplace transform, Journal of the AMS 10 p. 939-972 (1997). 
[KS01] _ Ind-sheaves, Astérisque 271136 pp. (2001).

[KS03] _ Microlocal study of ind-sheaves. I. Micro-support and regularity, Astérisque 284 p. 143-164 (2003).

[KS06] , Categories and sheaves, Grundlehren der Math. Wiss. 332 Springer-Verlag (2006).

[KS12] — Microlocal Euler classes and Hochschild homology, J. Inst. Math. Jussieu (2013).

math.arXiv:1203.4869

[KS12b] _ Deformation quantization modules, Astérisque Soc. Math. France. 345 (2012). arXiv:math.arXiv:1003.3304

[Ke10] K.S. Kedlaya, Good formal structures for flat meromorphic connections, I: Surfaces, Duke Math. J. 154 p. 343-418 (2010).

[Ke11] _ Good formal structures for flat meromorphic connections, II: Excellent schemes, J. Amer. Math. Soc. 24 p. 183-229 (2011).

[Lo61] S. Lojaciewicz, Sur le problème de la division, Studia Math. 8 pp. $87-$ 156, (1961).

[Ma67] B. Malgrange, Ideals of differentiable functions, Tata Institute, Oxford Univ. Press (1967).

[Mr67] A. Martineau, Théorèmes sur le prolongement analytique du type "Edge of the Wedge" Sem. Bourbaki, 340 (1967/68).

[Mo09] T. Mochizuki, Good formal structure for meromorphic flat connections on smooth projective surfaces, in Algebraic Analysis and around, Advances Studies in Pure Math. 54 Math. Soc. Japan p. 223-253 (2009).

[Mr09] G. Morando, Temperate holomorphic solutions of $\mathscr{D}$-modules on curves and formal invariants, Ann. Inst. Fourier (Grenoble), 59 (2009).

[Mr13] _ Constructibility of tempered solutions of holonomic Dmodules, preprint arXiv:1311.6621

[Na09] D. Nadler, Microlocal branes are constructible sheaves, Selecta Math. 15 p. 563619, (2009).

[NZ09] D. Nadler and E. Zaslow, Constructible sheaves and the Fukaya category, J. Amer. Math. Soc. 22 p. 233-286 (2009). 
[Pr08] L. Prelli, Sheaves on subanalytic sites, Rendiconti del Seminario Matematico dell'Universit di Padova, 120 p. 167-216 (2008).

[Pr12] _ Microlocalization of subanalytic sheaves, Mém. Soc. Math. France (2012).

[Ra08] A. C. Ramadoss, The relative Riemann-Roch theorem from Hochschild homology, New York J. Math. 14, p. 643-717 (2008), arXiv:math/0603127.

[Ra10] _ The Mukai pairing and integral transforms in Hochschild homology, Moscow Mathematical Journal, 10 p. 629-645 (2010).

[Sb12] C. Sabbah, Théorie de Hodge et correspondance de Hitchin-Kobayashi sauvage, d'après T. Mochizuki, Sém. Bourbaki 1050 (2011-12).

[Sa60] M. Sato, Theory of hyperfunctions, I \& II Journ. Fac. Sci. Univ. Tokyo, 8 139-193 487-436 (1959-1960).

[SGA4] S-G-A 4, Sém. Géom. Alg. (1963-64) by M. Artin, A. Grothendieck and J-L. Verdier, Théorie des topos et cohomologie étale des schémas, Lecture Notes in Math. 269, 270, 305 (1972/73).

[SKK73] M. Sato, T. Kawai and M. Kashiwara, Microfunctions and pseudodifferential equations, in Komatsu (ed.), Hyperfunctions and pseudodifferential equations, Proceedings Katata 1971, Lecture Notes in Math. Springer-Verlag 287 p. 265-529 (1973).

[Sc07] P. Schapira, Mikio Sato, a visionary of mathematics, Notices of the AMS, 54 Vol 2, (2007).

[Sc10] _ Triangulated categories for the analysts, in "Triangulated categories" London Math. Soc. LNS 375 Cambridge University Press, p. 371-389 (2010).

[ScSn94] P. Schapira and J-P. Schneiders, Index theorem for elliptic pairs, Astrisque Soc. Math. France 224 (1994)

[SSn13] P. Schapira and J.-P. Schneiders, Derived category of filtered objects, arXiv:math.AG:1306.1359

[Sn99] J.-P. Schneiders, Quasi-abelian categories and sheaves, Mém. Soc. Math. Fr. (N.S.) 76 (1999). 
[Sj82] J. Sjöstrand, Singularités analytiques microlocales, in Astérisque 95 Soc. Math. France (1982).

[VdB98] M. Van den Bergh, A relation between Hochschild homology and cohomology for Gorenstein rings, Proc. Amer. Math. Soc. 126 pp. 13451348 (1998). Erratum 130 p. 2809-2810 (2002).

Pierre Schapira

Institut de Mathématiques, Université Pierre et Marie Curie

and Mathematics Research Unit, University of Luxemburg

e-mail: schapira@math.jussieu.fr

http://www.math.jussieu.fr/ ${ }^{\sim}$ schapira/ 